\title{
Stability analysis and error estimates of local discontinuous Galerkin methods with implicit-explicit time-marching for nonlinear convection-diffusion problems
}

\author{
Haijin Wang ${ }^{\mathrm{a}}$, Chi-Wang Shu ${ }^{\mathrm{b}, *}$, Qiang Zhang ${ }^{\mathrm{a}}$ \\ ${ }^{a}$ Department of Mathematics, Nanjing University, Nanjing 210093, Jiangsu Province, \\ P. R. China. \\ ${ }^{b}$ Division of Applied Mathematics, Brown University, Providence, RI 02912, U.S.A.
}

\begin{abstract}
The main purpose of this paper is to analyze the stability and error estimates of the local discontinuous Galerkin (LDG) methods coupled with implicit-explicit (IMEX) time discretization schemes, for solving one-dimensional convectiondiffusion equations with a nonlinear convection. Both Runge-Kutta and multistep IMEX methods are considered. By the aid of the energy method, we show that the IMEX LDG schemes are unconditionally stable for the nonlinear problems, in the sense that the time-step $\tau$ is only required to be upper-bounded by a positive constant which depends on the flow velocity and the diffusion coefficient, but is independent of the mesh size $h$. We also give optimal error estimates for the IMEX LDG schemes, under the same temporal condition, if a monotone numerical flux is adopted for the convection. Numerical experiments are given to verify our main results.
\end{abstract}

Keywords: local discontinuous Galerkin method, implicit-explicit scheme, convection-diffusion equation, stability analysis, error estimate, energy method 2010 MSC: 65M12, 65M15, 65M60

\section{Introduction}

In this paper we extend our previous work 14] and continue to analyze the stability and error estimates of some fully discrete local discontinuous Galerkin (LDG) schemes coupled with implicit-explicit (IMEX) time discretization, for 5 solving a semilinear convection-diffusion problem with periodic boundary condition in one dimension.

\footnotetext{
Dedicated to Professor Claus-Dieter Munz on the occasion of his sixtieth birthday

* Corresponding author

Email addresses: hjwang@smail.nju.edu.cn (Haijin Wang), shu@dam.brown.edu (Chi-Wang Shu), qzh@nju.edu.cn (Qiang Zhang)
}

Preprint submitted to Journal of AMC

February 4, 2015

(C) 2015. This manuscript version is made available under the Elsevier user license http://www.elsevier.com/open-access/userlicense/1.0/ 
The LDG method was introduced by Cockburn and Shu for convectiondiffusion problems in [8] , motivated by the work of Bassi and Rebay [2] for compressible Navier-Stokes equations. As an extension of discontinuous Galerkin ${ }_{10}$ (DG) schemes for hyperbolic conservation laws [9], this scheme shares the advantages of the DG methods, such as good stability, high order accuracy, and flexibility on $h-p$ adaptivity and on complex geometry. Besides, a key advantage of this scheme is the local solvability, that is, the auxiliary variables approximating the gradient of the solution can be locally eliminated. Over the past

15 twenty years, there has been extensive study of the LDG methods for steady problems or in the semi-discrete framework, such as for elliptic problems [4], convection-diffusion problems [5], the Stokes system 7], the KdV type equations 18], Hamilton-Jacobi equations [17], time-dependent fourth order problems [10], and so on.

20 The time-discretization to the LDG method is necessary and should be studied carefully, in order to keep the above advantages and the original efficiency. In the fully-discrete framework, explicit Runge-Kutta time discretization methods were analyzed in [15]. This kind of time discretization is stable, efficient and accurate for solving convection-dominated convection-diffusion problems. How-

25 ever, for convection-diffusion equations which are not convection-dominated, explicit time discretization will suffer from a stringent time step restriction for stability [16]. When it comes to such problems, a natural consideration to overcome the small time step restriction is to use implicit time marching. Furthermore, in many applications the convection terms are often nonlinear, so hence it would be desirable to treat them explicitly while using implicit time discretization only for the linear diffusion terms. Such time discretizations are called implicit-explicit (IMEX) time discretizations [1]. Even for nonlinear diffusion terms, IMEX time discretizations would show their advantages in obtaining an elliptic algebraic system, which is easy to solve by many iterative methods.

35 If both convection and diffusion are treated implicitly, the resulting algebraic system will be far from elliptic and convergence of many iterative solvers will suffer.

In 14 we have discussed the LDG scheme coupled with three specific RungeKutta type IMEX schemes given in [1] and [3], for solving linear advection-

40 diffusion problems. We have shown that those schemes are unconditionally stable in the sense that the time step $\tau$ is only required to be upper bounded by a fixed constant which depends solely on the coefficients of advection and diffusion, but is independent of the mesh size. In this paper we will extend the above work to the nonlinear convection case, which is widely used in practice.

45 Also, we will apply our analysis technique to some multi-step IMEX schemes given in 11]. Furthermore, we would like to show the optimal error estimates for the convection-diffusion problem with a nonlinear convection, under the similar temporal condition as in the stability analysis, if a general monotone numerical flux is adopted.

We will mainly perform the error estimates of the LDG spatial discretization coupled with the third order IMEX RK time marching method given in [3] and the third order multi-step IMEX time marching method given in [1] for solving 
the nonlinear problems. As we pointed out in 14], there is one more stage for the explicit part than the implicit part in the third order IMEX RK scheme in 55 3], which makes the error analysis more complicated than the linear case 14, where we only considered the upwind numerical flux for the convection part, so that we can find a proper projection (Gauss-Radau projection) to eliminate the cell interface errors to obtain optimal error accuracy. While for the nonlinear problems, if we consider a general monotone numerical flux, it is difficult to find

60 such a projection to eliminate the cell interface errors, so we would like to resort to the technique used in [20] and 21] to carry out the error analysis for the third order IMEX LDG scheme. In the analysis, an a priori assumption is required, and the idea in 12] to divide the total error into spatial error and temporal error is also adopted. The error estimates for the first and second order IMEX

65 LDG schemes presented in 14 will not be presented in this paper to save space, because the numbers of explicit stages and implicit stages for these two lower order schemes are the same, hence their analysis is simpler than the third order scheme; see Remark 3

This paper is organized as follows. In Section 2 we present the semi-discrete 70 LDG scheme and some elemental conclusions about the finite element space as well as the LDG spatial discretization. In Section 3 we will present three specific IMEX RK fully-discrete LDG schemes for the model problem, and will concentrate on obtaining stability analysis and error estimate for the third order RK type IMEX LDG methods. Also, we present some multi-step IMEX LDG 75 schemes and their stability and error analysis in Section 4 In Section 5 we give numerical results to verify our results. The concluding remarks and some technical proofs are given in Section [6] and the Appendix, respectively.

\section{The LDG method}

In this section we present the definition of the semi-discrete LDG schemes for one dimensional nonlinear convection-diffusion problem

$$
U_{t}+f(U)_{x}-d U_{x x}=0, \quad(x, t) \in Q_{T}=(a, b) \times(0, T],
$$

with periodic boundary condition and the initial solution $U(x, 0)=U_{0}(x)$, where the diffusion coefficient $d>0$ is a constant, and $f$ is assumed to be differentiable.

\subsection{Discontinuous finite element space}

Let $\mathcal{T}_{h}=\left\{I_{j}=\left(x_{j-\frac{1}{2}}, x_{j+\frac{1}{2}}\right)\right\}_{j=1}^{N}$ be the partition of $\Omega=(a, b)$, where $x_{\frac{1}{2}}=a$ and $x_{N+\frac{1}{2}}=b$ are two boundary endpoints. Denote the cell length as $h_{j}=$ $x_{j+\frac{1}{2}}-x_{j-\frac{1}{2}}$ for $j=1, \ldots, N$, and define $h=\max _{j} h_{j}$. We assume $\mathcal{T}_{h}$ is quasi-

85 uniform in this paper, that is, there exists a positive constant $\nu$ such that for all $j$ there holds $h_{j} / h \geq \nu$, as $h$ goes to zero.

Associated with this mesh, we define the discontinuous finite element space

$$
V_{h}=\left\{v \in L^{2}(\Omega):\left.v\right|_{I_{j}} \in \mathcal{P}_{k}\left(I_{j}\right), \forall j=1, \ldots, N\right\},
$$


where $\mathcal{P}_{k}\left(I_{j}\right)$ denotes the space of polynomials in $I_{j}$ of degree at most $k \geq 1$. Furthermore, we would like to consider the (mesh-dependent) broken Sobolev space

$$
H^{1}\left(\mathcal{T}_{h}\right)=\left\{\phi \in L^{2}(\Omega):\left.\phi\right|_{I_{j}} \in H^{1}\left(I_{j}\right), \forall j=1, \ldots, N\right\},
$$

which contains the discontinuous finite element space $V_{h}$. Note that the functions in this space are allowed to have discontinuities across element interfaces. At each element interface point, for any piecewise function $p$, there are two 90 traces along the right-hand and left-hand, denoted by $p^{+}$and $p^{-}$, respectively. As usual, the jump is denoted by $\llbracket p \rrbracket=p^{+}-p^{-}$.

Now we present the following inverse properties with respect to the finite element space $V_{h}$, where the standard norms and semi-norms in Sobolev spaces are used. For any function $v \in V_{h}$, there exists a positive constant $\mu>0$ independent of $v, h$ and $j$ such that

$$
\begin{aligned}
& \left\|v_{x}\right\|_{I_{j}} \leq \mu h^{-1}\|v\|_{I_{j}}, \\
& \|v\|_{\infty, I_{j}} \leq \mu h^{-1 / 2}\|v\|_{I_{j}}, \\
& \|v\|_{\partial I_{j}} \leq \sqrt{\mu h^{-1}}\|v\|_{I_{j}},
\end{aligned}
$$

where $\|v\|_{\partial I_{j}}=\sqrt{\left(v_{j-\frac{1}{2}}^{+}\right)^{2}+\left(v_{j+\frac{1}{2}}^{-}\right)^{2}}$ is the $L^{2}$-norm on the boundary of $I_{j}$. We call $\mu$ the inverse constant and denote $\|v\|_{\Gamma_{h}}=\left(\sum_{j=1}^{N}\|v\|_{\partial I_{j}}^{2}\right)^{1 / 2}$.

In this paper we will use two Gauss-Radau projections, from $H^{1}\left(\mathcal{T}_{h}\right)$ to $V_{h}$, denoted by $\pi_{h}^{-}$and $\pi_{h}^{+}$respectively. For any function $p \in H^{1}\left(\mathcal{T}_{h}\right)$, the projection $\pi_{h}^{ \pm} p$ is defined as the unique element in $V_{h}$ such that, in each element $I_{j}=\left(x_{j-\frac{1}{2}}, x_{j+\frac{1}{2}}\right)$

$$
\begin{array}{lll}
\left(\pi_{h}^{-} p-p, v\right)_{I_{j}}=0 & \forall v \in \mathcal{P}_{k-1}\left(I_{j}\right), & \left(\pi_{h}^{-} p\right)_{j+\frac{1}{2}}^{-}=p_{j+\frac{1}{2}}^{-} \\
\left(\pi_{h}^{+} p-p, v\right)_{I_{j}}=0 & \forall v \in \mathcal{P}_{k-1}\left(I_{j}\right), & \left(\pi_{h}^{+} p\right)_{j-\frac{1}{2}}^{+}=p_{j-\frac{1}{2}}^{+}
\end{array}
$$

Denote by $\eta=p-\pi_{h}^{ \pm} p$ the projection error. By a standard scaling argument [6] , it is easy to obtain the following approximation property

$$
\|\eta\|_{I_{j}}+h^{1 / 2}\|\eta\|_{\infty, I_{j}}+h^{1 / 2}\|\eta\|_{\partial I_{j}} \leq C h^{\min (k+1, s)}\|p\|_{H^{s}\left(I_{j}\right)}, \quad \forall j,
$$

where the bounding constant $C>0$ is independent of $h$ and $j$.

In what follows we will mainly use the inverse inequalities 2.4 and the approximation property (2.6) in global form by summing up the above local inequalities over $j=1,2, \ldots, N$. The conclusions are almost the same as their local versions, so they are omitted here.

\subsection{Semi-discrete $L D G$ scheme}

Following [8], we introduce the auxiliary variable $Q=\sqrt{d} U_{x}$, and find the approximation solutions in the discontinuous finite element space, based on the following equivalent first-order differential system

$$
U_{t}+(f(U)-\sqrt{d} Q)_{x}=0, \quad Q+(-\sqrt{d} U)_{x}=0, \quad(x, t) \in Q_{T},
$$


with the same initial condition and the periodic boundary condition.

The semi-discrete LDG scheme is defined as follows: for any $t>0$, find the numerical solution $(u(x, t), q(x, t)) \in V_{h} \times V_{h}$, such that, for any test functions $\boldsymbol{z}=(v, r) \in V_{h} \times V_{h}$, the variation forms

$$
\begin{aligned}
\left(u_{t}, v\right)_{j} & =\mathcal{H}_{j}(f(u), v)-\sqrt{d} \mathcal{L}_{j}^{+}(q, v), \\
(q, r)_{j} & =-\sqrt{d} \mathcal{L}_{j}^{-}(u, r),
\end{aligned}
$$

hold in each cell $I_{j}, j=1,2, \ldots, N$. Here and below we drop the arguments $x$ and $t$ if there is no confusion. Here $(\cdot, \cdot)_{j}$ is the usual inner product in $L^{2}\left(I_{j}\right)$ and

$$
\begin{aligned}
\mathcal{H}_{j}(f(u), v) & =\left(f(u), v_{x}\right)_{j}-\hat{f}(u)_{j+\frac{1}{2}} v_{j+\frac{1}{2}}^{-}+\hat{f}(u)_{j-\frac{1}{2}} v_{j-\frac{1}{2}}^{+}, \\
\mathcal{L}_{j}^{ \pm}(v, r) & =\left(v, r_{x}\right)_{j}-v_{j+\frac{1}{2}}^{ \pm} r_{j+\frac{1}{2}}^{-}+v_{j-\frac{1}{2}}^{ \pm} r_{j-\frac{1}{2}}^{+},
\end{aligned}
$$

where $\hat{f}(u)=\hat{f}\left(u^{-}, u^{+}\right)$is a monotone numerical flux, which is Lipschitz continuous, consistent with $f(u)$, nondecreasing and nonincreasing with respect to its first and second arguments, respectively. Note that the alternating numerical flux [8] is adopted in (2.9b).

For the convenience of analysis, we sum up the variational formulations (2.8) over all cells. Then we can write the semi-discrete LDG scheme in the global form: for any $t>0$, find the numerical solution $(u, q) \in V_{h} \times V_{h}$ such that the variation equations

$$
\begin{aligned}
\left(u_{t}, v\right) & =\mathcal{H}(f(u), v)+\mathcal{L}(q, v), \\
(q, r) & =\mathcal{K}(u, r),
\end{aligned}
$$

hold for any $\boldsymbol{z}=(v, r) \in V_{h} \times V_{h}$, where $(\cdot, \cdot)=\sum_{j=1}^{N}(\cdot, \cdot)_{j}$ is the inner product in $L^{2}(\Omega)$, and

$$
\mathcal{H}=\sum_{j=1}^{N} \mathcal{H}_{j}, \quad \mathcal{L}=-\sqrt{d} \sum_{j=1}^{N} \mathcal{L}_{j}^{+}, \quad \mathcal{K}=-\sqrt{d} \sum_{j=1}^{N} \mathcal{L}_{j}^{-} .
$$

The initial condition $u(x, 0)$ can be taken as any approximation of the given initial solution $U_{0}(x)$, for example, the local Gauss-Radau projection (2.5) of $U_{0}(x)$. We have now defined the semi-discrete LDG scheme.

\subsection{Properties of the $L D G$ spatial discretization}

In this subsection, we will give several lemmas to illustrate some properties of the LDG spatial discretization, which will be used many times in this paper.

First we would like to consider the linear part. Lemma 2.1 demonstrates the skew symmetric property of the operators $\mathcal{L}$ and $\mathcal{K}$, and Lemma 2.2 results from the definitions of $\mathcal{L}^{ \pm}$and the Gauss-Radau projection. The proofs are straightforward, so we skip the details. For the readers who are interested in the proof, we refer to 19]. Lemma 2.3 given in 14] builds up an important relationship between the auxiliary variable and the prime variable, which plays a key role in the stability analysis. 
Lemma 2.1. For any $w, v \in H^{1}\left(\mathcal{T}_{h}\right)$, there holds $\mathcal{L}(w, v)=-\mathcal{K}(v, w)$.

Lemma 2.2. For any $w \in H^{1}\left(\mathcal{T}_{h}\right)$ and $v \in V_{h}$, there holds $\mathcal{L}^{ \pm}\left(\pi_{h}^{ \pm} w-w, v\right)=0$.

Lemma 2.3. Suppose $\boldsymbol{w}=(u, q) \in V_{h} \times V_{h}$ satisfy (2.10b), then

$$
\left\|u_{x}\right\|+\sqrt{\mu h^{-1}}\|u\| \leq \frac{C_{\mu}}{\sqrt{d}}\|q\|,
$$

where the bounding constant $C_{\mu}$ is independent of $h$ and $d$.

Next we consider the nonlinear part, namely, $\mathcal{H}$, and present two conclusions. Lemma 2.4 is from [13]. Lemma 2.5 gives two boundedness properties. To derive Lemma 2.5 we would like to assume that the consistent numerical flux $\hat{f}$ is Lipschitz continuous with respect to each component, and denote the Lipschitz constant as $C_{f}$. Then we have

$$
\left|\hat{f}\left(p_{1}^{-}, p_{1}^{+}\right)-\hat{f}\left(p_{2}^{-}, p_{2}^{+}\right)\right| \leq C_{f}\left(\left|p_{1}^{-}-p_{2}^{-}\right|+\left|p_{1}^{+}-p_{2}^{+}\right|\right), \quad \forall p_{1}, p_{2},
$$

which implies

$$
\left|f^{\prime}(p)\right| \leq C_{f}, \quad \forall p
$$

if $f$ is differentiable. This assumption is acceptable, if we focus on the error estimate to smooth solutions.

Remark 1. In this paper, we would like to use the notation $C_{f}$ to denote the generic positive constant, which is independent of the mesh size $h$ and the time step $\tau$. Each occurrence may have a different value.

Lemma 2.4. For any $v \in H^{1}\left(\mathcal{T}_{h}\right)$, there holds $\mathcal{H}(f(v), v) \leq 0$.

In this paper, we will use the notation $\mathcal{D}(u, w ; v)$ to represent the difference between $\mathcal{H}(f(u), v)$ and $\mathcal{H}(f(w), v)$ for arbitrary $u, w \in H^{1}\left(\mathcal{T}_{h}\right)$ and $v \in V_{h}$, i.e,

$$
\mathcal{D}(u, w ; v)=\mathcal{H}(f(u), v)-\mathcal{H}(f(w), v) .
$$

Lemma 2.5. For any $u, w, v \in V_{h}$, there hold the following inequalities

$$
\begin{aligned}
& |\mathcal{H}(f(u), v)| \leq C_{f}\left(\left\|u_{x}\right\|+\sqrt{\mu h^{-1}}\|u\|\right)\|v\|, \\
& |\mathcal{D}(u, w ; v)| \leq C_{f}\|u-w\|\left(\left\|v_{x}\right\|+\sqrt{\mu h^{-1}}\|v\|\right) .
\end{aligned}
$$

if (2.13) holds.

Proof. From (2.9a) and the periodic boundary condition, we have

$$
\begin{aligned}
\mathcal{H}(f(u), v) & =\sum_{j=1}^{N}\left\{\left(f(u), v_{x}\right)_{j}-\hat{f}(u)_{j+\frac{1}{2}} v_{j+\frac{1}{2}}^{-}+\hat{f}(u)_{j-\frac{1}{2}} v_{j-\frac{1}{2}}^{+}\right\} \\
& =\sum_{j=1}^{N}\left\{\left(f(u), v_{x}\right)_{j}+\hat{f}(u)_{j-\frac{1}{2}} \llbracket v \rrbracket_{j-\frac{1}{2}}\right\} .
\end{aligned}
$$


Integrating by parts yields

$$
\begin{aligned}
\mathcal{H}(f(u), v) & =\sum_{j=1}^{N}\left\{-\left(f(u)_{x}, v\right)_{j}+f\left(u_{j+\frac{1}{2}}^{-}\right) v_{j+\frac{1}{2}}^{-}-f\left(u_{j-\frac{1}{2}}^{+}\right) v_{j-\frac{1}{2}}^{+}+\hat{f}(u)_{j-\frac{1}{2}} \llbracket v \rrbracket_{j-\frac{1}{2}}\right\} \\
& =\sum_{j=1}^{N}\left\{-\left(f^{\prime}(u) u_{x}, v\right)_{j}+f\left(u_{j-\frac{1}{2}}^{-}\right) v_{j-\frac{1}{2}}^{-}-f\left(u_{j-\frac{1}{2}}^{+}\right) v_{j-\frac{1}{2}}^{+}+\hat{f}(u)_{j-\frac{1}{2}} \llbracket v \rrbracket_{j-\frac{1}{2}}\right\} \\
& =\sum_{j=1}^{N}\left(\theta_{1}^{j}+\theta_{2}^{j}+\theta_{3}^{j}+\theta_{4}^{j}\right) .
\end{aligned}
$$

Noting that

$$
\begin{aligned}
\theta_{2}^{j}+\theta_{3}^{j}+\theta_{4}^{j} & =\left(f\left(u^{-}\right)-f\left(u^{+}\right)\right) v^{-}-f\left(u^{+}\right) \llbracket v \rrbracket+\hat{f}(u) \llbracket v \rrbracket \\
& =-f^{\prime}\left(\sigma_{1}\right) \llbracket u \rrbracket v^{-}+\left(\hat{f}\left(u^{-}, u^{+}\right)-\hat{f}\left(u^{+}, u^{+}\right)\right) \llbracket v \rrbracket,
\end{aligned}
$$

where the last equality follows from the mean-value theorem, with $\sigma_{1}=a_{1} u^{-}+$ $\left(1-a_{1}\right) u^{+}$for some $a_{1} \in[0,1]$, and the consistency of the numerical flux $\hat{f}$. Here we have dropped the subscript $j-\frac{1}{2}$ for simplicity of notation. By the Lipschitz continuity of $\hat{f}$, we have

$$
\left|\hat{f}\left(u^{-}, u^{+}\right)-\hat{f}\left(u^{+}, u^{+}\right)\right| \leq C_{f}|\llbracket u \rrbracket| .
$$

Then using the inverse inequality (2.4c) we have

$$
\left|\theta_{2}^{j}+\theta_{3}^{j}+\theta_{4}^{j}\right| \leq C_{f} \sqrt{\mu h^{-1}}\left|\llbracket u \rrbracket_{j-\frac{1}{2}}\right|\left(\|v\|_{I_{j-1}}+\|v\|_{I_{j}}\right) .
$$

Moreover, the simple use of Cauchy-Schwarz inequality and 2.13b gives rise to

$$
\left|\theta_{1}^{j}\right| \leq C_{f}\left\|u_{x}\right\|_{I_{j}}\|v\|_{I_{j}}
$$

As a result, summing the above two estimates over $j=1, \cdots, N$, we get 2.15).

Next we will prove (2.16). From (2.17) we have

$\mathcal{D}(u, w ; v)=\sum_{j=1}^{N}\left\{\left(f(u)-f(w), v_{x}\right)_{j}+(\hat{f}(u)-\hat{f}(w))_{j-\frac{1}{2}} \llbracket v \rrbracket_{j-\frac{1}{2}}\right\}=\sum_{j=1}^{N}\left(\theta_{5}^{j}+\theta_{6}^{j}\right)$.

It follows from the mean-value theorem and the Cauchy-Schwarz inequality that

$$
\left|\theta_{5}^{j}\right|=\left|\left(f^{\prime}\left(\sigma_{2}\right)(u-w), v_{x}\right)_{j}\right| \leq C_{f}\|u-w\|_{I_{j}}\left\|v_{x}\right\|_{I_{j}}
$$

where $\sigma_{2}=a_{2} u+\left(1-a_{2}\right) w$ for some $a_{2} \in[0,1]$. From 2.13a we get

$$
\left|\theta_{6}^{j}\right| \leq C_{f}\left(\left|u^{-}-w^{-}\right|_{j-\frac{1}{2}}+\left|u^{+}-w^{+}\right|_{j-\frac{1}{2}}\right)\left|\llbracket v \rrbracket_{j-\frac{1}{2}}\right| .
$$

Thus by the inverse inequality (2.4c) we have

$$
\left|\theta_{6}^{j}\right| \leq C_{f} \sqrt{\mu h^{-1}}\left(\|u-w\|_{I_{j-1}}+\|u-w\|_{I_{j}}\right)\left|\llbracket v \rrbracket_{j-\frac{1}{2}}\right| .
$$

As a consequence, by summing (2.22) and (2.23) over $j=1, \cdots, N$, we obtain (2.16). 


\section{IMEX RK LDG schemes}

In this paper we would like to consider the LDG spatial discretization coupled with three specific IMEX Runge-Kutta schemes up to third order which are presented in [14].

\subsection{Fully discrete schemes}

Let $\left\{t^{n}=n \tau\right\}_{n=0}^{M}$ be the uniform partition of the time interval $[0, T]$, with time step $\tau$. The time step could actually change from step to step, but in this paper we take the time step as a constant for simplicity. Given $u^{n}$, hence $\left(u^{n}, q^{n}\right)$, we would like to find the numerical solution at the next time level $t^{n+1}$, (maybe through several intermediate stages $t^{n, \ell}$ ), by the following IMEX RK methods.

The LDG scheme with the first order IMEX time-marching scheme, where the convection part is treated by the forward Euler method and the diffusion part is treated by the backward Euler method, is given in the following form:

$$
\begin{aligned}
\left(u^{n+1}, v\right) & =\left(u^{n}, v\right)+\tau \mathcal{H}\left(f\left(u^{n}\right), v\right)+\tau \mathcal{L}\left(q^{n+1}, v\right), \\
\left(q^{n+1}, r\right) & =\mathcal{K}\left(u^{n+1}, r\right),
\end{aligned}
$$

for any function $(v, r) \in V_{h} \times V_{h}$.

The LDG scheme with the second order IMEX time marching scheme given in 1 is:

$$
\begin{aligned}
\left(u^{n, 1}, v\right)= & \left(u^{n}, v\right)+\gamma \tau \mathcal{H}\left(f\left(u^{n}\right), v\right)+\gamma \tau \mathcal{L}\left(q^{n, 1}, v\right) \\
\left(u^{n+1}, v\right)= & \left(u^{n}, v\right)+\delta \tau \mathcal{H}\left(f\left(u^{n}\right), v\right)+(1-\delta) \tau \mathcal{H}\left(f\left(u^{n, 1}\right), v\right) \\
& +(1-\gamma) \tau \mathcal{L}\left(q^{n, 1}, v\right)+\gamma \tau \mathcal{L}\left(q^{n+1}, v\right) \\
\left(q^{n, \ell}, r\right)= & \mathcal{K}\left(u^{n, \ell}, r\right), \quad \ell=1,2
\end{aligned}
$$

for any function $(v, r) \in V_{h} \times V_{h}$, where $\gamma=1-\frac{\sqrt{2}}{2}$ and $\delta=1-\frac{1}{2 \gamma}$. Here $w^{n, 2}=w^{n+1}$.

The LDG scheme with the third order IMEX time marching scheme proposed in [3] reads: for any function $(v, r) \in V_{h} \times V_{h}$,

$$
\begin{aligned}
\left(u^{n, \ell}, v\right) & =\left(u^{n}, v\right)+\tau \sum_{i=0}^{3}\left(a_{\ell i} \mathcal{H}\left(f\left(u^{n, i}\right), v\right)+\hat{a}_{\ell i} \mathcal{L}\left(q^{n, i}, v\right)\right), \quad \text { for } \quad \ell=1,2,3 \\
\left(u^{n+1}, v\right) & =\left(u^{n}, v\right)+\tau \sum_{i=0}^{3}\left(b_{i} \mathcal{H}\left(f\left(u^{n, i}\right), v\right)+\hat{b}_{i} \mathcal{L}\left(q^{n, i}, v\right)\right), \\
\left(q^{n, \ell}, r\right) & =\mathcal{K}\left(u^{n, \ell}, r\right), \quad \text { for } \quad \ell=1,2,3
\end{aligned}
$$


where the coefficients are given in the following table

\begin{tabular}{|c|cccc||cccc|c|}
\hline \multirow{3}{*}{$a_{\ell i}$} & $\frac{1+\gamma}{2}-\alpha_{1}$ & $\alpha_{1}$ & 0 & 0 & 0 & $\frac{1-\gamma}{2}$ & $\gamma$ & 0 & $\hat{a}_{\ell i}$ \\
& 0 & $1-\alpha_{2}$ & $\alpha_{2}$ & 0 & 0 & $\beta_{1}$ & $\beta_{2}$ & $\gamma$ & \\
\hline$b_{i}$ & 0 & $\beta_{1}$ & $\beta_{2}$ & $\gamma$ & 0 & $\beta_{1}$ & $\beta_{2}$ & $\gamma$ & $\hat{b}_{i}$ \\
\hline
\end{tabular}

${ }_{145}$ The left half of the table lists $a_{\ell i}$ and $b_{i}$, with the columns from left to right corresponding to $i=0,1,2,3$, and the first three rows from top to bottom corresponding to $\ell=1,2,3$. Similarly, the right half lists $\hat{a}_{\ell i}$ and $\hat{b}_{i}$. In (3.4), $\gamma \approx 0.435866521508459$, is the middle root of $6 x^{3}-18 x^{2}+9 x-1=0$. Also, $\beta_{1}=-\frac{3}{2} \gamma^{2}+4 \gamma-\frac{1}{4}, \beta_{2}=\frac{3}{2} \gamma^{2}-5 \gamma+\frac{5}{4}$. The parameter $\alpha_{1}$ is chosen as -0.35 in [3] and $\alpha_{2}=\frac{\frac{1}{3}-2 \gamma^{2}-2 \beta_{2} \alpha_{1} \gamma}{\gamma(1-\gamma)}$.

In the next two subsections we will consider the stability analysis and error estimates for the above schemes. From Lemmas 2.4 and 2.5 we can see that, for the nonlinear convection term, the operator $\mathcal{H}$ also has the similar properties as in the linear case. Hence conceptually we can get the same stability property 55 for the nonlinear problems as what we got in 14] along similar arguments.

Actually, the procedure for the first and second order schemes are almost the same as the one for the linear problem in 14], the only difference is: for the nonlinear problem, we need to use property 2.16 to deal with the convection terms generated by the difference of two different time levels. Furthermore, the error estimate for the first and second order schemes can be easily obtained along the similar line as the stability analysis. The idea will also be used for the third order scheme. However, for the third order scheme, since there is one more explicit stage than the implicit stage, the procedure is different and much more technical than the linear case. Hence in the following, we pay our attention to the third order time-marching, namely, the scheme (3.3).

\subsection{Stability analysis}

As we mentioned before, the procedure to deal with the linear convection terms in 14 cannot be used to handle the nonlinear convection terms directly. Because in the linear case, besides the terms which are the differences of solu170 tions at two different time levels, there are also terms which are convex combinations of solutions at three time levels, for example, $\mathcal{H}\left(u^{n, 2}-2 u^{n, 1}+u^{n}, v\right)$. The estimate for such terms is easy in the linear case, but if we change $u^{n, \ell}$ into $f\left(u^{n, \ell}\right)$, we cannot find a similar estimate. In such nonlinear case we would need to divide this term into $\mathcal{D}\left(u^{n, 2}, u^{n, 1} ; v\right)-\mathcal{D}\left(u^{n, 1}, u^{n} ; v\right)$ and then use the prop175 erty (2.16) to estimate it. Also, there is a negative (non-positive) term $\mathcal{H}\left(u^{n, 2}-\right.$ $\left.2 u^{n, 1}, u^{n, 2}-2 u^{n, 1}\right)$ in the linear case, but $\mathcal{H}\left(f\left(u^{n, 2}\right)-2 f\left(u^{n, 1}\right), u^{n, 2}-2 u^{n, 1}\right)$ is not necessarily negative. Therefore, we would need to treat the nonlinear convection part in a different manner.

Theorem 3.1. There exists a positive constant $\tau_{0}$ which is proportional to $d / C_{f}^{2}$ but is independent of $h$, such that if $\tau \leq \tau_{0}$, then the solution of scheme (3.3) 
satisfies

$$
\left\|u^{n}\right\| \leq\left\|u^{0}\right\|, \quad \forall n
$$

if (2.13) holds.

Proof. For the convenience of analysis, we follow 14 to introduce a series of notations

$$
\begin{aligned}
& \mathbb{E}_{1} w^{n}=w^{n, 1}-w^{n}, \quad \mathbb{E}_{2} w^{n}=w^{n, 2}-2 w^{n, 1}+w^{n}, \\
& \mathbb{E}_{3} w^{n}=2 w^{n, 3}+w^{n, 2}-3 w^{n, 1}, \quad \mathbb{E}_{4} w^{n}=w^{n+1}-w^{n, 3},
\end{aligned}
$$

for arbitrary $w$, and rewrite the scheme (3.3) into the following compact form

$$
\begin{aligned}
\left(\mathbb{E}_{\ell} u^{n}, v\right) & =\Phi_{\ell}\left(\boldsymbol{u}^{n}, v\right)+\Psi_{\ell}\left(\boldsymbol{q}^{n}, v\right), \quad \text { for } \quad \ell=1,2,3,4, \\
\left(q^{n, \ell}, r\right) & =\mathcal{K}\left(u^{n, \ell}, r\right), \quad \text { for } \quad \ell=1,2,3
\end{aligned}
$$

where $\boldsymbol{u}^{n}=\left(u^{n}, u^{n, 1}, u^{n, 2}, u^{n, 3}\right)$ and $\boldsymbol{q}^{n}=\left(q^{n, 1}, q^{n, 2}, q^{n, 3}\right)$, and

$$
\begin{aligned}
& \Phi_{\ell}\left(\boldsymbol{u}^{n}, v\right)=\sum_{i=0}^{3} \delta_{\ell i} \tau \mathcal{H}\left(f\left(u^{n, i}\right), v\right) \\
& \Psi_{\ell}\left(\boldsymbol{q}^{n}, v\right)=\theta_{\ell 1} \tau \mathcal{L}\left(q^{n, 1}, v\right)+\theta_{\ell 2} \tau \mathcal{L}\left(q^{n, 2}-2 q^{n, 1}, v\right)+\theta_{\ell 3} \tau \mathcal{L}\left(q^{n, 3}, v\right)
\end{aligned}
$$

180

for $\ell=1,2,3,4$. The coefficients $\delta_{\ell i}$ and $\theta_{\ell i}$ are listed in Table 1 See 14] for more details.

Table 1: The coefficients $\delta_{\ell i}$ and $\theta_{\ell i}$ in 3.8 and 3.9.

\begin{tabular}{|c|c|c|c|c|c|c|c|}
\hline & \multicolumn{5}{|c|}{$\delta_{\ell i}$} & \multicolumn{3}{|c|}{$\theta_{\ell i}$} \\
\hline$\ell i$ & 0 & 1 & 2 & 3 & 1 & 2 & 3 \\
\hline 1 & $\gamma$ & 0 & 0 & 0 & $\gamma$ & 0 & 0 \\
\hline 2 & $\frac{1-3 \gamma}{2}-\alpha_{1}$ & $\alpha_{1}$ & 0 & 0 & $\frac{1-\gamma}{2}$ & $\gamma$ & 0 \\
\hline 3 & $\frac{1-5 \gamma}{2}-\alpha_{1}$ & $2\left(1-\alpha_{2}\right)+\alpha_{1}$ & $2 \alpha_{2}$ & 0 & $2\left(\frac{9}{4}-\frac{11}{4} \gamma-\beta_{1}\right)$ & $2\left(1-\beta_{1}-\frac{\gamma}{2}\right)$ & $2 \gamma$ \\
\hline 4 & 0 & $\alpha_{2}-\beta_{2}-\gamma$ & $\beta_{2}-\alpha_{2}$ & $\gamma$ & 0 & 0 & 0 \\
\hline
\end{tabular}

Along the same line as the proof in [14], we take the test functions $v=$ $u^{n, 1}, u^{n, 2}-2 u^{n, 1}, u^{n, 3}$ and $2 u^{n+1}$ in (3.7), for $\ell=1,2,3,4$, respectively. Adding them together, we get the energy equation

$$
\left\|u^{n+1}\right\|^{2}-\left\|u^{n}\right\|^{2}+\mathcal{S}=\mathcal{T}_{\mathrm{c}}+\mathcal{T}_{\mathrm{d}}
$$

where

$$
\mathcal{S}=\frac{1}{2}\left(\left\|\mathbb{E}_{1} u^{n}\right\|^{2}+\left\|\mathbb{E}_{2} u^{n}\right\|+\left\|\mathbb{E}_{31} u^{n}\right\|^{2}+\left\|\mathbb{E}_{32} u^{n}\right\|^{2}+2\left\|\mathbb{E}_{4} u^{n}\right\|^{2}\right)
$$


is the stability coming from the time-marching, with $\mathbb{E}_{31} u^{n}=u^{n, 3}+u^{n, 2}-2 u^{n, 1}$ and $\mathbb{E}_{32} u^{n}=u^{n, 3}-u^{n, 1}$. Furthermore,

$$
\begin{aligned}
& \mathcal{T}_{\mathrm{c}}=\Phi_{1}\left(\boldsymbol{u}^{n}, u^{n, 1}\right)+\Phi_{2}\left(\boldsymbol{u}^{n}, u^{n, 2}-2 u^{n, 1}\right)+\Phi_{3}\left(\boldsymbol{u}^{n}, u^{n, 3}\right)+2 \Phi_{4}\left(\boldsymbol{u}^{n}, u^{n+1}\right), \\
& \mathcal{T}_{\mathrm{d}}=\Psi_{1}\left(\boldsymbol{q}^{n}, u^{n, 1}\right)+\Psi_{2}\left(\boldsymbol{q}^{n}, u^{n, 2}-2 u^{n, 1}\right)+\Psi_{3}\left(\boldsymbol{q}^{n}, u^{n, 3}\right)+2 \Psi_{4}\left(\boldsymbol{q}^{n}, u^{n+1}\right),
\end{aligned}
$$

are given in the same form as in 14]. We will consider them one by one.

Denote $\mathbf{w}^{\top}=\left(q^{n, 1}, q^{n, 2}-2 q^{n, 1}, q^{n, 3}\right)$, and $\|\mathbf{w}\|^{2}=\left\|q^{n, 1}\right\|^{2}+\left\|q^{n, 2}-2 q^{n, 1}\right\|^{2}+$ $\left\|q^{n, 3}\right\|^{2}$. Noting that

$$
\mathcal{L}\left(q_{1}, u_{2}\right)=-\mathcal{K}\left(u_{2}, q_{1}\right)=-\left(q_{2}, q_{1}\right)=-\left(q_{1}, q_{2}\right),
$$

holds for any pairs $\left(u_{1}, q_{1}\right)$ and $\left(u_{2}, q_{2}\right)$, owing to Lemma 2.1 and (2.10b). By using (3.12) we get

$$
\mathcal{T}_{\mathrm{d}}=-\tau \int_{\Omega} \mathbf{w}^{\top} \mathbb{A} \mathbf{w} \mathrm{d} x
$$

where

$$
\mathbb{A}=\frac{1}{2}\left(\begin{array}{ccc}
2 \theta_{11} & \theta_{21} & \theta_{31} \\
\theta_{21} & 2 \theta_{22} & \theta_{32} \\
\theta_{31} & \theta_{32} & 2 \theta_{33}
\end{array}\right) .
$$

Since all the principal minor determinants of $\mathbb{A}$ are positive, we can conclude that $\mathbb{A}$ is positive definite. In fact we can show in the same way that

$$
\mathcal{T}_{\mathrm{d}} \leq-\frac{\gamma}{4} \tau\|\mathbf{w}\|^{2} \leq 0
$$

The estimate to the remaining term $\mathcal{T}_{\mathrm{c}}$ is a little more complicated, due to the nonlinearity of the flux. From (3.8) and after some algebraic manipulations we get

$$
\mathcal{T}_{\mathrm{c}}=\mathcal{T}_{\mathrm{c}}^{(1)}+\mathcal{T}_{\mathrm{c}}^{(2)}+\mathcal{T}_{\mathrm{c}}^{(3)}
$$

where

$$
\begin{aligned}
\mathcal{T}_{\mathrm{c}}^{(1)}= & \tau\left(\delta_{10}-\delta_{20}-\delta_{21}\right) \mathcal{H}\left(f\left(u^{n, 1}\right), u^{n, 1}\right)+\tau \sum_{i=0}^{2} \delta_{3 i} \mathcal{H}\left(f\left(u^{n, 3}\right), u^{n, 3}\right), \\
\mathcal{T}_{\mathrm{c}}^{(2)}= & \tau\left(\delta_{20}+\delta_{21}\right) \mathcal{H}\left(f\left(u^{n, 1}\right), u^{n, 2}-u^{n, 1}\right)+\tau \sum_{i=1}^{3} 2 \delta_{4 i} \mathcal{H}\left(f\left(u^{n, i}\right), u^{n+1}-u^{n, 3}\right), \\
\mathcal{T}_{\mathrm{c}}^{(3)}= & -\tau \delta_{10} \mathcal{D}\left(u^{n, 1}, u^{n} ; u^{n, 1}\right)-\tau \delta_{20} \mathcal{D}\left(u^{n, 1}, u^{n} ; u^{n, 2}-2 u^{n, 1}\right) \\
& -\tau \delta_{30} \mathcal{D}\left(u^{n, 1}, u^{n} ; u^{n, 3}\right)+\tau\left(\delta_{32}+2 \delta_{42}\right) \mathcal{D}\left(u^{n, 2}, u^{n, 1} ; u^{n, 3}\right) \\
& +\tau\left(2 \delta_{43}-\sum_{i=0}^{2} \delta_{3 i}\right) \mathcal{D}\left(u^{n, 3}, u^{n, 1} ; u^{n, 3}\right) .
\end{aligned}
$$


It is easy to check that both coefficients $\left(\delta_{10}-\delta_{20}-\delta_{21}\right)$ and $\sum_{i=0}^{2} \delta_{3 i}$ in (3.17a) are positive, so owing to Lemma 2.4 we have $\mathcal{T}_{\mathrm{c}}^{(1)} \leq 0$. Noticing that all ${ }_{185}$ the coefficients in the expressions of $\mathcal{T}_{\mathrm{c}}^{(2)}$ and $\mathcal{T}_{\mathrm{c}}^{(3)}$ are bounded, we will utilize (2.15) and (2.16) to estimate $\mathcal{T}_{\mathrm{c}}^{(2)}$ and $\mathcal{T}_{\mathrm{c}}^{(3)}$, respectively.

For example, if we denote the first and the second term of $\mathcal{T}_{\mathrm{c}}^{(2)}$ by $V_{1}$ and $V_{2}$, respectively, then from (2.15), Lemma 2.3 and the triangle inequality we get

$$
\begin{aligned}
V_{1} & \left.\leq C_{f} \tau\left(\left\|u_{x}^{n, 1}\right\|+\sqrt{\mu h^{-1}}\right) \llbracket u^{n, 1} \rrbracket\right)\left\|u^{n, 2}-u^{n, 1}\right\| \\
& \leq \frac{C_{f} C_{\mu}}{\sqrt{d}} \tau\left\|q^{n, 1}\right\|\left\|u^{n, 2}-u^{n, 1}\right\| \leq \frac{C_{f} C_{\mu}}{\sqrt{d}} \tau\left\|q^{n, 1}\right\|\left(\left\|\mathbb{E}_{2} u^{n}\right\|+\left\|\mathbb{E}_{1} u^{n}\right\|\right),
\end{aligned}
$$

and

$$
\begin{aligned}
V_{2} & \left.\leq C_{f} \tau \sum_{\ell=1}^{3}\left(\left\|u_{x}^{n, \ell}\right\|+\sqrt{\mu h^{-1}}\right) \llbracket u^{n, \ell} \rrbracket\right)\left\|\mathbb{E}_{4} u^{n}\right\| \leq \frac{C_{f} C_{\mu}}{\sqrt{d}} \tau \sum_{\ell=1}^{3}\left\|q^{n, \ell}\right\|\left\|\mathbb{E}_{4} u^{n}\right\| \\
& \leq \frac{C_{f} C_{\mu}}{\sqrt{d}} \tau\left(\left\|q^{n, 1}\right\|+\left\|q^{n, 2}-2 q^{n, 1}\right\|+\left\|q^{n, 3}\right\|\right)\left\|\mathbb{E}_{4} u^{n}\right\| .
\end{aligned}
$$

Similarly, from (2.16), Lemma 2.3 and the triangle inequality we have

$$
\mathcal{T}_{\mathrm{c}}^{(3)} \leq \frac{C_{f} C_{\mu}}{\sqrt{d}} \tau\left(\left\|\mathbb{E}_{1} u^{n}\right\|+\left\|\mathbb{E}_{2} u^{n}\right\|+\left\|\mathbb{E}_{32} u^{n}\right\|\right)\left(\left\|q^{n, 1}\right\|+\left\|q^{n, 2}-2 q^{n, 1}\right\|+\left\|q^{n, 3}\right\|\right) .
$$

Hence we can derive

$$
\mathcal{T}_{\mathrm{c}} \leq \frac{C_{f} C_{\mu}}{\sqrt{d}} \tau \mathcal{S}^{\frac{1}{2}}\|\mathbf{w}\| \leq \varepsilon \tau\|\mathbf{w}\|^{2}+\frac{C_{f}^{2} C_{\mu}^{2}}{4 \varepsilon d} \tau \mathcal{S},
$$

by using Young's inequality, where $\varepsilon>0$ is arbitrary. Noting that the value of $C_{f}$ may be several times of the value $C_{f}$ in (2.15) and (2.16), but we use the same notation as we mentioned in Remark 1 just for the sake of simplicity.

We would like to take $\varepsilon=\frac{\gamma}{4}$, then owing to (3.10), (3.15) and (3.18) we have

$$
\left\|u^{n+1}\right\|^{2}-\left\|u^{n}\right\|^{2}+\mathcal{S} \leq \frac{C_{f}^{2} C_{\mu}^{2}}{d \gamma} \tau \mathcal{S},
$$

Thus $\left\|u^{n+1}\right\| \leq\left\|u^{n}\right\|$, if $\tau \leq \tau_{0}=\frac{d \gamma}{C_{f}^{2} C_{\mu}^{2}}$. This completes the proof of this theorem.

\subsection{Error estimate}

In this subsection we would like to obtain the optimal error estimate. To this end, we would like to assume that the exact solution $U(x, t)$ is sufficiently smooth, for example,

$$
U \in L^{\infty}\left(0, T ; H^{k+2}\right), \quad D_{t} U \in L^{\infty}\left(0, T ; H^{k+1}\right), \quad \text { and } \quad D_{t}^{4} U \in L^{\infty}\left(0, T ; L^{2}\right),
$$


where $D_{t}^{\ell} U$ is the $\ell$-th order time derivative of $U$, and $L^{\infty}\left(0, T ; H^{s}(D)\right)$ represents the set of functions $v$ such that $\max _{0 \leq t \leq T}\|v(\cdot, t)\|_{H^{s}(D)}<\infty$.

Furthermore, we would like to assume the flux function $f$ in (2.1) is smooth enough, for example, $f \in C^{k+3}$. In particular, we would like to assume

$$
\left|f^{\prime}(p)\right|,\left|f^{\prime \prime}(p)\right| \leq C_{f}
$$

for arbitrary $p \in \mathbb{R}$. For a given initial condition, this assumption is reasonable with the original or a suitably modified flux $f$, due to the maximum principle; see [20] for more details.

The main result is stated in the following theorem.

Theorem 3.2. Let $u$ be the numerical solution of scheme (3.3). The finite element space $V_{h}$ is the space of piecewise polynomials with degree $k>1$ on the quasi-uniform triangulations of $\Omega=(a, b)$. Let $U(x, t)$ be the exact solution of problem (2.1) which satisfies the smoothness assumption (3.20), then there exist positive constants $h_{0}$ and $\tau_{0}$, such that if $h \leq h_{0}$ and $\tau \leq \tau_{0}$, then there holds the following error estimate

$$
\max _{n \tau \leq T}\left\|U\left(x, t^{n}\right)-u^{n}\right\| \leq C\left(h^{k+1}+\tau^{3}\right),
$$

where $T$ is the final computing time and the bounding constant $C>0$ is independent of $h$ and $\tau$.

The proof of this theorem is lengthy and technical. We split this process into three steps.

\section{Step 1: reference functions}

Based on the idea of [12], we would like to let $U^{0}=U_{0}(x)$, and define $\left(U^{n, \ell}(x), Q^{n, \ell}(x)\right)$ as the solution of the following third order IMEX time discrete problem:

$$
\begin{aligned}
U^{n, \ell} & =U^{n}+\tau \sum_{i=0}^{3}\left(-a_{\ell i} f\left(U^{n, i}\right)_{x}+\hat{a}_{\ell i} \sqrt{d}\left(Q^{n, i}\right)_{x}\right), \quad \text { for } \quad \ell=1,2,3, \\
U^{n+1} & =U^{n}+\tau \sum_{i=0}^{3}\left(-b_{i} f\left(U^{n, i}\right)_{x}+\hat{b}_{i} \sqrt{d}\left(Q^{n, i}\right)_{x}\right)
\end{aligned}
$$

for arbitrary $n \tau \leq T$, where we have dropped the argument $x$ for simplicity of notation, the coefficients $a_{\ell i}, \hat{a}_{\ell i}$ and $b_{i}, \hat{b}_{i}$ were given in (3.4), and

$$
Q^{n, \ell}=\sqrt{d}\left(U^{n, \ell}\right)_{x}, \quad \text { for } \quad \ell=1,2,3 .
$$

Since $D_{t}^{4} U \in L^{\infty}\left(0, T ; L^{2}\right)$, we can get that the error between the exact solution and the time discrete solution of (3.23) is bounded in the form

$$
\left\|U\left(x, t^{n}\right)-U^{n}(x)\right\| \leq C \tau^{3}, \quad \forall n \tau \leq T,
$$


where $C$ only depends on the final computing time $T$. Furthermore, we can follow the similar line as the proof of Theorem 3.1 in [12] to prove that

$$
\left\|U^{n, \ell}\right\|_{H^{k+1}} \leq C, \quad\left\|Q^{n, \ell}\right\|_{H^{k+1}} \leq C, \quad \text { and } \quad\left\|\mathbb{E}_{\ell+1} U^{n}\right\|_{H^{k+1}} \leq C \tau,
$$

for any $n$ and $\ell=0,1,2,3$ under consideration, if the exact solution $U(x, t) \in$

$H^{k+2}, U_{t}(x, t) \in H^{k+1}$, and the flux function $f$ is assumed to be in $C^{k+3}$. We omit the detailed proof to save space.

It is easy to verify that these reference functions satisfy the following variational forms

$$
\begin{aligned}
\left(\mathbb{E}_{\ell} U^{n}, v\right) & =\Phi_{\ell}\left(\boldsymbol{U}^{n}, v\right)+\Psi_{\ell}\left(\boldsymbol{Q}^{n}, v\right), \quad \text { for } \quad \ell=1,2,3,4, \\
\left(Q^{n, \ell}, r\right) & =\mathcal{K}\left(U^{n, \ell}, r\right), \quad \text { for } \quad \ell=1,2,3
\end{aligned}
$$

for any $(v, r) \in V_{h} \times V_{h}$, which is very analogous to scheme (3.7). Here we denote $\boldsymbol{U}^{n}=\left(U^{n}, U^{n, 1}, U^{n, 2}, U^{n, 3}\right)$ and $\boldsymbol{Q}^{n}=\left(Q^{n, 1}, Q^{n, 2}, Q^{n, 3}\right)$.

Step 2: error presentation, error equations and energy equations

For the purpose of estimating the error between the exact solution and the numerical solution $U\left(x, t^{n}\right)-u^{n}$, we only need to estimate $U^{n}-u^{n}$, the error between the solution of (3.23) and the numerical solution.

Towards this end, at each time stage we denote the error between the time discrete solution and the numerical solution by

$$
e^{n, \ell}=\left(e_{u}^{n, \ell}, e_{q}^{n, \ell}\right)=\left(U^{n, \ell}-u^{n, \ell}, Q^{n, \ell}-q^{n, \ell}\right) .
$$

As the standard treatment in finite element analysis, we would like to divide the error in the form $\boldsymbol{e}^{n, \ell}=\boldsymbol{\xi}^{n, \ell}-\boldsymbol{\eta}^{n, \ell}$, where

$$
\begin{aligned}
& \boldsymbol{\xi}^{n, \ell}=\left(\xi_{u}^{n, \ell}, \xi_{q}^{n, \ell}\right)=\left(\pi_{h}^{-} U^{n, \ell}-u^{n, \ell}, \pi_{h}^{+} Q^{n, \ell}-q^{n, \ell}\right), \\
& \boldsymbol{\eta}^{n, \ell}=\left(\eta_{u}^{n, \ell}, \eta_{q}^{n, \ell}\right)=\left(\pi_{h}^{-} U^{n, \ell}-U^{n, \ell}, \pi_{h}^{+} Q^{n, \ell}-Q^{n, \ell}\right) .
\end{aligned}
$$

It follows from the linearity of projections $\pi_{h}^{ \pm}$and (2.6) that the stage projection errors and their evolutions satisfy

$$
\begin{aligned}
& \left\|\eta_{u}^{n, \ell}\right\|+h^{1 / 2}\left\|\eta_{u}^{n, \ell}\right\|_{\Gamma_{h}}+h^{1 / 2}\left\|\eta_{u}^{n, \ell}\right\|_{\infty}+\left\|\eta_{q}^{n, \ell}\right\| \leq C h^{k+1}, \\
& \left\|\mathbb{E}_{\ell+1} \eta_{u}^{n}\right\|+h^{1 / 2}\left\|\mathbb{E}_{\ell+1} \eta_{u}^{n}\right\|_{\Gamma_{h}} \leq C h^{k+1} \tau
\end{aligned}
$$

where the bounding constant $C>0$ depends solely on the smoothness of the solution $U^{n, \ell}$ and $Q^{n, \ell}$, which was given in (3.25), independent of $n, h$ and $\tau$.

In what follows we will focus on the estimate to the error in the finite element space. Towards the goal of obtaining the final estimate for $\xi_{u}^{n}$, we would like first to establish the energy equation, following the similar line as the previous stability analysis.

We subtracting the variational forms in (3.26) from those in scheme (3.7), at the same order. Since the projection error related terms in $\Psi_{\ell}$ and $\mathcal{K}$ vanish 
by Lemma 2.2 we obtain the following error equations

$$
\begin{aligned}
\left(\mathbb{E}_{\ell} \xi_{u}^{n}, v\right) & =\Phi_{\ell}\left(\boldsymbol{U}^{n}, v\right)-\Phi_{\ell}\left(\boldsymbol{u}^{n}, v\right)+\Psi_{\ell}\left(\boldsymbol{\xi}_{\boldsymbol{q}}^{n}, v\right)+\left(\mathbb{E}_{\ell} \eta_{u}^{n}, v\right), \quad \text { for } \quad \ell=1,2,3,4, \\
\left(\xi_{q}^{n, \ell}, r\right) & =\mathcal{K}\left(\xi_{u}^{n, \ell}, r\right)+\left(\eta_{q}^{n, \ell}, r\right), \quad \text { for } \quad \ell=1,2,3,
\end{aligned}
$$

where $\boldsymbol{\xi}_{\boldsymbol{q}}^{n}=\left(\xi_{q}^{n, 1}, \xi_{q}^{n, 2}, \xi_{q}^{n, 3}\right)$.

Taking $v=\xi_{u}^{n, 1}, \xi_{u}^{n, 2}-2 \xi_{u}^{n, 1}, \xi_{u}^{n, 3}$ and $2 \xi_{u}^{n+1}$ in (3.29a), for $\ell=1,2,3,4$ respectively, and adding them together, we obtain the energy equation

$$
\left\|\xi_{u}^{n+1}\right\|^{2}-\left\|\xi_{u}^{n}\right\|^{2}+\tilde{\mathcal{S}}=\tilde{\mathcal{T}}_{\mathrm{c}}+\tilde{\mathcal{T}}_{\mathrm{d}}+\tilde{\mathcal{T}}_{\mathrm{p}}
$$

where

$$
\tilde{\mathcal{S}}=\frac{1}{2}\left(\left\|\mathbb{E}_{1} \xi_{u}^{n}\right\|^{2}+\left\|\mathbb{E}_{2} \xi_{u}^{n}\right\|+\left\|\mathbb{E}_{31} \xi_{u}^{n}\right\|^{2}+\left\|\mathbb{E}_{32} \xi_{u}^{n}\right\|^{2}+2\left\|\mathbb{E}_{4} \xi_{u}^{n}\right\|^{2}\right),
$$

and

$\tilde{\mathcal{T}}_{\mathrm{c}}=\sum_{\ell=1}^{4}\left(\Phi_{\ell}\left(\boldsymbol{U}^{n}, v_{\ell}\right)-\Phi_{\ell}\left(\boldsymbol{u}^{n}, v_{\ell}\right)\right), \quad \tilde{\mathcal{T}}_{\mathrm{d}}=\sum_{\ell=1}^{4} \Psi_{\ell}\left(\boldsymbol{\xi}_{\boldsymbol{q}}^{n, \ell}, v_{\ell}\right), \quad \tilde{\mathcal{T}}_{\mathrm{p}}=\sum_{\ell=1}^{4}\left(\mathbb{E}_{\ell} \eta_{u}^{n}, v_{\ell}\right)$

Here and below we use the simplified notations

$$
v_{1}=\xi_{u}^{n, 1}, v_{2}=\xi_{u}^{n, 2}-2 \xi_{u}^{n, 1}, v_{3}=\xi_{u}^{n, 3} \text {, and } v_{4}=2 \xi_{u}^{n+1} .
$$

\section{Step 3: energy analysis}

To derive the final error estimates, we would like to follow 21] and make the a priori assumption for $m$, if $m \tau \leq T$ :

$$
\left\|e_{u}^{n, \ell}\right\|_{\infty} \leq C_{0} h, \quad \text { for } \quad n \leq m, \ell=0,1,2,3,
$$

where $C_{0}$ is a positive constant independent of $m, h, \tau$ and $u$.

Lemma 3.3. Let $U^{n, \ell}$ be the solution of (3.23) satisfying the smoothness (3.25), and $u^{n, \ell}$ be the solution of scheme (3.3), then for arbitrary $v \in V_{h}$ we have

$$
\left|\mathcal{D}\left(U^{n, \ell}, u^{n, \ell} ; v\right)\right| \leq C_{f}\left(\left\|\xi_{u}^{n, \ell}\right\|+h^{k+1}\right)\left(\left\|v_{x}\right\|+\sqrt{\mu h^{-1}}\|v\|\right) .
$$

Proof. The proof for this lemma is similar to that for (2.16). The projection error approximation property (3.28a ) is used in the proof. We skip the details to save space.

Lemma 3.4. There exist positive constant $C$ independent of $n, h, \tau$, and $\tau_{0}$ independent of $h$, such that, if $\tau \leq \tau_{0}$, then

$$
\left\|\xi_{u}^{n+1}\right\|^{2}-\left\|\xi_{u}^{n}\right\|^{2} \leq C \tau \sum_{\ell=0}^{4}\left\|\xi_{u}^{n, \ell}\right\|^{2}+C\left(h^{2 k+2} \tau+h^{2 k} \tau^{4}\right)
$$

${ }_{225}$ if the a priori assumption (3.32) holds, where $\xi_{u}^{n, 0}=\xi_{u}^{n}$ and $\xi_{u}^{n, 4}=\xi_{u}^{n+1}$. 
Proof. We only need to estimate the three terms on the right-hand side of the energy equation (3.30). A simple use of the Cauchy-Schwarz inequality, the Young's inequality and (3.28b yields

$$
\tilde{\mathcal{T}}_{\mathrm{p}} \leq \tau \sum_{\ell=1}^{4}\left\|\xi_{u}^{n, \ell}\right\|^{2}+\frac{C}{\tau} \sum_{\ell=1}^{4}\left\|\mathbb{E}_{\ell} \eta_{u}^{n}\right\|^{2} \leq \tau \sum_{\ell=1}^{4}\left\|\xi_{u}^{n, \ell}\right\|^{2}+C h^{2 k+2} \tau .
$$

The estimate for $\tilde{\mathcal{T}}_{\mathrm{d}}$ is similar to the one for $\mathcal{T}_{\mathrm{d}}$ in the stability analysis. Similar to the property (3.12) we have

$$
\mathcal{L}\left(\xi_{q}^{1}, \xi_{u}^{2}\right)=-\mathcal{K}\left(\xi_{u}^{2}, \xi_{q}^{1}\right)=-\left(\xi_{q}^{2}, \xi_{q}^{1}\right)+\left(\eta_{q}^{2}, \xi_{q}^{1}\right),
$$

for any pairs of $\left(\xi_{u}^{1}, \xi_{q}^{1}\right)$ and $\left(\xi_{u}^{2}, \xi_{q}^{2}\right)$, due to Lemma 2.1 and (3.29b). By using property (3.36) and the Cauchy-Schwarz inequality, the Young's inequality and (3.28a), we can derive, along the similar line as the estimate for $\mathcal{T}_{\mathrm{d}}$ (3.11c),

$$
\tilde{\mathcal{T}}_{\mathrm{d}} \leq-\left(\frac{\gamma}{4}-\varepsilon\right) \tau\|\tilde{\mathbf{w}}\|^{2}+C_{\varepsilon} h^{2 k+2} \tau,
$$

for arbitrary $\varepsilon>0$, where $C_{\varepsilon}$ is a positive constant only depending on $\varepsilon$, and

$$
\|\tilde{\mathbf{w}}\|^{2}=\left\|\xi_{q}^{n, 1}\right\|^{2}+\left\|\xi_{q}^{n, 2}-2 \xi_{q}^{n, 1}\right\|^{2}+\left\|\xi_{q}^{n, 3}\right\|^{2} .
$$

The procedure of estimating $\tilde{\mathcal{T}}_{\mathrm{c}}$ is more complicated than the one for estimating $\mathcal{T}_{\mathrm{c}}$ in the stability analysis. From (3.8) we have $\tilde{\mathcal{T}}_{\mathrm{c}}=Z_{1}+Z_{2}$, where

$$
\begin{aligned}
& Z_{1}=\tau \sum_{\ell=1}^{3} \sum_{i=0}^{3} \delta_{\ell i} \mathcal{D}\left(U^{n, i}, u^{n, i} ; v_{\ell}\right)+2 \tau \sum_{i=0}^{3} \delta_{4 i} \mathcal{D}\left(U^{n, i}, u^{n, i} ; v_{3}\right), \\
& Z_{2}=2 \tau \sum_{i=0}^{3} \delta_{4 i} \mathcal{D}\left(U^{n, i}, u^{n, i} ; \mathbb{E}_{4} \xi_{u}^{n}\right) .
\end{aligned}
$$

Let us consider them one by one. A direct use of Lemma 3.3 gives rise to

$$
Z_{1} \leq C_{f} \tau \sum_{\ell=0}^{3}\left(\left\|\xi_{u}^{n, \ell}\right\|+h^{k+1}\right) R
$$

where

$$
R=\sum_{i=1}^{3}\left(\left\|\left(v_{i}\right)_{x}\right\|+\sqrt{\mu h^{-1}} \llbracket v_{i} \rrbracket\right) .
$$

Noticing that for any pair of $\left(\xi_{u}^{n, \ell}, \xi_{q}^{n, \ell}\right)$ there holds

$$
\left\|\left(\xi_{u}^{n, \ell}\right)_{x}\right\|+\sqrt{\mu h^{-1}}\left\|\xi_{u}^{n, \ell}\right\| \leq \frac{C_{\mu}}{\sqrt{d}}\left(\left\|\xi_{q}^{n, \ell}\right\|+\left\|\eta_{q}^{n, \ell}\right\|\right),
$$


which is similar to (2.12) and the proof is also similar. Moreover, by the linear structure of (3.29b), (3.39) also holds for any linear combination of any pairs of $\left(\xi_{u}^{n, \ell}, \xi_{q}^{n, \ell}\right)$, for example, for $v_{2}=\xi_{u}^{n, 2}-2 \xi_{u}^{n, 1}$, we have

$$
\left\|\left(v_{2}\right)_{x}\right\|+\sqrt{\mu h^{-1}}\left\|v_{2}\right\| \leq \frac{C_{\mu}}{\sqrt{d}}\left(\left\|\xi_{q}^{n, 2}-2 \xi_{q}^{n, 1}\right\|+\left\|\eta_{q}^{n, 2}-2 \eta_{q}^{n, 1}\right\|\right) .
$$

Hence by (3.39) and (3.28a) we have

$$
R \leq \frac{C_{\mu}}{\sqrt{d}}\left(\left\|\xi_{q}^{n, 1}\right\|+\left\|\xi_{q}^{n, 2}-2 \xi_{q}^{n, 1}\right\|+\left\|\xi_{q}^{n, 3}\right\|+h^{k+1}\right) .
$$

Then a simple use of the Young's inequality yields

$$
Z_{1} \leq \frac{C_{f}^{2} C_{\mu}^{2}}{d} \tau \sum_{\ell=0}^{3}\left\|\xi_{u}^{n, \ell}\right\|^{2}+\varepsilon \tau\|\tilde{\mathbf{w}}\|^{2}+\left(\frac{C_{f}^{2} C_{\mu}^{2}}{d}+\frac{C_{f} C_{\mu}}{\sqrt{d}}\right) h^{2 k+2} \tau,
$$

for arbitrary $\varepsilon>0$. Here $C_{f}$ depends on $\varepsilon$. To obtain the sharp estimate for $Z_{2}$, we would like to start with the following identity

$$
\begin{aligned}
Z_{2}= & 2 \delta_{42} \tau\left[\mathcal{D}\left(U^{n, 2}, u^{n, 2} ; \mathbb{E}_{4} \xi_{u}^{n}\right)-\mathcal{D}\left(U^{n, 1}, u^{n, 1} ; \mathbb{E}_{4} \xi_{u}^{n}\right)\right] \\
& +2 \delta_{43} \tau\left[\mathcal{D}\left(U^{n, 3}, u^{n, 3} ; \mathbb{E}_{4} \xi_{u}^{n}\right)-\mathcal{D}\left(U^{n, 1}, u^{n, 1} ; \mathbb{E}_{4} \xi_{u}^{n}\right)\right],
\end{aligned}
$$

where the differences of reference functions play critical roles. By the aid of an important quantity $\alpha(\cdot, \cdot)$ introduced in 21] which measures the numerical viscosity, and after a long and technical analysis, we can derive

$$
\begin{aligned}
Z_{2} \leq & C \tau \sum_{\ell=1}^{3}\left\|\xi_{u}^{n, \ell}\right\|^{2}+\varepsilon \tau\|\tilde{\mathbf{w}}\|^{2}+C\left(h^{2 k+2} \tau+h^{2 k} \tau^{4}\right) \\
& +\left(C_{f}^{2} \tau+C_{f}^{2} C_{0}^{2} \tau+\frac{C_{f}^{2} C_{\mu}^{2}}{d} \tau+\varepsilon\right)\left\|\mathbb{E}_{4} \xi_{u}^{n}\right\|^{2},
\end{aligned}
$$

for arbitrary $\varepsilon>0$. The definition of $\alpha(\cdot, \cdot)$ and the detailed proof are left to the Appendix, and we continue to obtain the final error estimate.

We choose $\varepsilon$ small enough and let $\tau \leq \tau_{0}$, where $\tau_{0}$ is a positive constant independent of $h$, such that the coefficient in front of $\left\|\mathbb{E}_{4} \xi_{u}^{n}\right\|^{2}$ is not over $1 / 2$. Then from (3.40) and (3.42) we have

$$
\mathcal{T}_{\mathrm{c}}^{\prime} \leq C \tau \sum_{\ell=0}^{3}\left\|\xi_{u}^{n, \ell}\right\|^{2}+2 \varepsilon \tau\|\tilde{\mathbf{w}}\|^{2}+\frac{1}{2}\left\|\mathbb{E}_{4} \xi_{u}^{n}\right\|^{2}+C\left(h^{2 k+2} \tau+h^{2 k} \tau^{4}\right) .
$$

Consequently, by (3.30), (3.35), (3.37) and (3.43) we have

$$
\left\|\xi_{u}^{n+1}\right\|^{2}-\left\|\xi_{u}^{n}\right\|^{2} \leq C \tau \sum_{\ell=0}^{4}\left\|\xi_{u}^{n, \ell}\right\|^{2}-\left(\frac{\gamma}{4}-3 \varepsilon\right) \tau\|\tilde{\mathbf{w}}\|^{2}+C\left(h^{2 k+2} \tau+h^{2 k} \tau^{4}\right) .
$$

Hence, we are led to the desired result (3.34) if $\varepsilon \leq \frac{\gamma}{12}$. 
The estimate for the intermediate stage values $\left\|\xi_{u}^{n, \ell}\right\|$ emerging in (3.34) can be obtained using a similar argument as the proof for Lemma 3.4 So we omit the details and only state it in the following lemma.

Lemma 3.5. There exist positive constant $C$ independent of $n, h, \tau$, and $\tau_{0}$ independent of $h$, such that, if $\tau \leq \tau_{0}$, then

$$
\left\|\xi_{u}^{n, \ell}\right\|^{2} \leq C\left(\left\|\xi_{u}^{n}\right\|^{2}+h^{2 k+2} \tau\right), \quad \text { for } \quad \ell=1,2,3 .
$$

Combining Lemmas 3.4 and 3.5 and by the aid of the discrete Gronwall's inequality, we can derive

$$
\left\|\xi_{u}^{n}\right\|^{2} \leq e^{C n \tau}\left(\left\|\xi_{u}^{0}\right\|^{2}+h^{2 k+2}+h^{2 k} \tau^{3}\right),
$$

if $\tau \leq \tau_{0}$. Noting that $\xi_{u}^{0}=0$, then we have

$$
\left\|\xi_{u}^{n}\right\| \leq C\left(h^{k+1}+h^{k} \tau^{3 / 2}\right), \quad \forall n \tau \leq T .
$$

Before arriving at the final error estimate, we need to verify that the a priori assumption (3.32) is reasonable. We will use the method of induction, along the similar argument line as that in 21]. Since $\xi_{u}^{0}=0$, the approximation property (3.28a implies $\left\|e_{u}^{0}\right\|_{\infty} \leq C_{0} h$, where $C_{0}$ only depends on the smoothness of the initial solution $U_{0}(x)$. Also, Lemma 3.5 shows $\left\|\xi_{u}^{0, \ell}\right\| \leq C h^{k+1} \tau^{1 / 2}$ for $\ell=1,2,3$, then by the triangle inequality, the inverse inequality (2.4b) and the approximation property 3.28a we have

$$
\left\|e_{u}^{0, \ell}\right\|_{\infty} \leq \mu h^{-1 / 2}\left\|\xi_{u}^{0, \ell}\right\|+\left\|\eta_{u}^{0, \ell}\right\|_{\infty} \leq C \mu h^{k+1 / 2} \tau^{1 / 2}+C h^{k+1 / 2} \leq C_{0} h,
$$

for $k>1$. Assuming (3.32) holds for $m$, we can show it also holds for $m+1$. From (3.46) we know $\left\|\xi_{u}^{m+1}\right\| \leq C\left(h^{k+1}+h^{k} \tau^{3 / 2}\right) \leq C h^{2}$, if $k>1$. Hence

$$
\left\|e_{u}^{m+1}\right\|_{\infty} \leq \mu h^{-1 / 2}\left\|\xi_{u}^{m+1}\right\|+\left\|\eta_{u}^{m+1}\right\|_{\infty} \leq C \mu h^{3 / 2}+C h^{k+1 / 2} \leq C h^{3 / 2}
$$

and for $\ell=1,2,3$, by Lemma 3.5 we can also deduce that

$$
\left\|e_{u}^{m+1, \ell}\right\|_{\infty} \leq C h^{3 / 2} .
$$

Apparently there exists a positive constant $h_{0}<1$ such that $C h^{1 / 2} \leq C_{0}$, if $h \leq h_{0}$, consequently we get $\left\|e_{u}^{m+1, \ell}\right\| \leq C_{0} h$, for $\ell=0,1,2,3$. Thus the assumption (3.32) is reasonable.

As a consequence, by Young's inequality we have

$$
\left\|\xi_{u}^{n}\right\| \leq C\left(h^{k+1}+h^{2 k}+\tau^{3}\right) \leq C\left(h^{k+1}+\tau^{3}\right),
$$

235 if $h \leq h_{0}$. Finally, owing to (3.24), (3.28a), 3.47) and the triangle inequality we get the main error estimate stated in Theorem 3.2

Remark 2. The requirement $k>1$ is not necessary in the numerical experiments, it is only for the theoretical consideration. Since our analysis relies on the a priori error assumption (3.32), and to ensure this assumption, we have to demand $k>1$. 
Remark 3. We can also prove the optimal error accuracy for the first and second order schemes (3.1) and (3.2), along the similar argument line as shown in this section, but it is not necessary to make the a priori assumption (3.32), since in the lower order IMEX schemes, the number of explicit stages is the same as the number of the implicit stages [14, hence no trouble term like $Z_{2}$ (see (3.42) ) will appear in the analysis. The optimal error accuracy can be obtained for arbitrary $k \geq 1$ in the first and second order case, under the same temporal condition as that in Theorem 3.2

\section{Multi-step IMEX LDG schemes}

In this section, we will study the stability property and error estimates of multi-step IMEX schemes coupled with the LDG spatial discretization. We would like to consider the second and third order schemes given by Gottlieb and Wang [11].

The second order multi-step IMEX scheme, coupled with LDG method, has the following form: for any $(v, r) \in V_{h} \times V_{h}$, we have

$$
\begin{aligned}
\left(u^{n+1}, v\right)= & \left(u^{n}, v\right)+\frac{3}{2} \tau \mathcal{H}\left(f\left(u^{n}\right), v\right)-\frac{1}{2} \tau \mathcal{H}\left(f\left(u^{n-1}\right), v\right) \\
& +\frac{3}{4} \tau \mathcal{L}\left(q^{n+1}, v\right)+\frac{1}{4} \tau \mathcal{L}\left(q^{n-1}, v\right) .
\end{aligned}
$$

for $n \geq 1$. As for the auxiliary variable $q^{n}$, we have the same variational form as before:

$$
\left(q^{n}, r\right)=\mathcal{K}\left(u^{n}, r\right), \quad \forall n .
$$

For arbitrary $(v, r) \in V_{h} \times V_{h}$, the LDG scheme with the third order multistep IMEX time marching method has the following variational form:

$$
\begin{aligned}
\left(u^{n+1}, v\right)= & \left(u^{n}, v\right)+\frac{23}{12} \tau \mathcal{H}\left(f\left(u^{n}\right), v\right)-\frac{4}{3} \tau \mathcal{H}\left(f\left(u^{n-1}\right), v\right)+\frac{5}{12} \tau \mathcal{H}\left(f\left(u^{n-2}\right), v\right) \\
& +\frac{2}{3} \tau \mathcal{L}\left(q^{n+1}, v\right)+\frac{5}{12} \tau \mathcal{L}\left(q^{n-1}, v\right)-\frac{1}{12} \tau \mathcal{L}\left(q^{n-3}, v\right),
\end{aligned}
$$

for $n \geq 3$, and for $q^{n}$, the variational form is still given by 4.1b).

In the following two subsections, we will proceed to the stability analysis and error estimates for the above multi-step IMEX LDG schemes. Since the analysis for the second one and third order cases is similar, we only give the proof for the third order scheme (4.2) as an example. Similar results can also be obtained for the fourth order scheme given in [11], but we omit the details to save space.

\subsection{Stability analysis}

Theorem 4.1. There exists a positive constant $\tau_{0}$ only depending on $d / C_{f}^{2}$ but independent of $h$, such that if $\tau \leq \tau_{0}$, then the solution of the scheme (4.1) 
satisfies

$$
\left\|u^{n+1}\right\|^{2}+\tau \sum_{j=1}^{n}\left\|q^{j+1}\right\|^{2} \leq C \sum_{j=0}^{1}\left(\left\|u^{j}\right\|^{2}+\tau\left\|q^{j}\right\|^{2}\right), \quad \text { for } \quad n \geq 1
$$

if (2.13) holds. Also, under the same condition, the solution of scheme (4.2) satisfies

$$
\left\|u^{n+1}\right\|^{2}+\tau \sum_{j=3}^{n}\left\|q^{j+1}\right\|^{2} \leq C \sum_{j=0}^{3}\left(\left\|u^{j}\right\|+\tau\left\|q^{j}\right\|^{2}\right), \quad \text { for } \quad n \geq 3
$$

where the bounding constant $C$ is independent of $h$ and $\tau$.

Proof. We only give the proof for (4.4), and (4.3) can be easily obtained along the similar line. Taking $v=u^{n+1}$ in (4.2) we obtain

$$
\frac{1}{2}\left\|u^{n+1}\right\|^{2}+\frac{1}{2}\left\|u^{n+1}-u^{n}\right\|^{2}-\frac{1}{2}\left\|u^{n}\right\|^{2}=T_{1}+T_{2},
$$

where after some manipulation and by Lemma 2.4 we have

$$
\begin{aligned}
T_{1}= & \tau \mathcal{H}\left(f\left(u^{n+1}\right), u^{n+1}\right)-\tau \mathcal{D}\left(u^{n+1}, u^{n} ; u^{n+1}\right)+\frac{11}{12} \tau \mathcal{D}\left(u^{n}, u^{n-1} ; u^{n+1}\right) \\
& -\frac{5}{12} \tau \mathcal{D}\left(u^{n-1}, u^{n-2} ; u^{n+1}\right) \\
\leq & \tau\left[-\mathcal{D}\left(u^{n+1}, u^{n} ; u^{n+1}\right)+\frac{11}{12} \mathcal{D}\left(u^{n}, u^{n-1} ; u^{n+1}\right)-\frac{5}{12} \mathcal{D}\left(u^{n-1}, u^{n-2} ; u^{n+1}\right)\right],
\end{aligned}
$$

and from the property (3.12) we can get

$$
\begin{aligned}
T_{2} & =\tau \mathcal{L}\left(\frac{2}{3} q^{n+1}+\frac{5}{12} q^{n-1}-\frac{1}{12} q^{n-3}, u^{n+1}\right) \\
& =-\frac{2}{3} \tau\left\|q^{n+1}\right\|^{2}-\frac{5}{12} \tau\left(q^{n+1}, q^{n-1}\right)+\frac{1}{12} \tau\left(q^{n+1}, q^{n-3}\right) .
\end{aligned}
$$

Summing in time leads to

$$
\begin{aligned}
& \frac{1}{2}\left\|u^{n+1}\right\|^{2}-\frac{1}{2}\left\|u^{3}\right\|^{2}+\frac{1}{2} \sum_{j=3}^{n}\left\|u^{j+1}-u^{j}\right\|^{2}+\frac{2}{3} \tau \sum_{j=3}^{n}\left\|q^{j+1}\right\|^{2} \\
= & \tau \sum_{j=3}^{n}\left[-\mathcal{D}\left(u^{j+1}, u^{j} ; u^{j+1}\right)+\frac{11}{12} \mathcal{D}\left(u^{j}, u^{j-1} ; u^{j+1}\right)-\frac{5}{12} \mathcal{D}\left(u^{j-1}, u^{j-2} ; u^{j+1}\right)\right] \\
& -\frac{5}{12} \tau \sum_{j=3}^{n}\left(q^{j+1}, q^{j-1}\right)+\frac{1}{12} \tau \sum_{j=3}^{n}\left(q^{j+1}, q^{j-3}\right)=\text { RHS. }
\end{aligned}
$$

Exploiting (2.16) and Lemma 2.3 for the three terms in the first line, for example

$$
\begin{aligned}
\left|\mathcal{D}\left(u^{j+1}-u^{j}, u^{j+1}\right)\right| & \leq C_{f}\left\|u^{j+1}-u^{j}\right\|\left(\left\|u_{x}^{j+1}\right\|+\sqrt{\mu h^{-1}}\left\|u^{j+1}\right\|\right) \\
& \leq \frac{C_{f} C_{\mu}}{\sqrt{d}}\left\|u^{j+1}-u^{j}\right\|\left\|q^{j+1}\right\|,
\end{aligned}
$$


and applying the Cauchy-Schwarz inequality for the two terms in the second line, we get

$$
\begin{aligned}
R H S \leq & \frac{C_{f} C_{\mu} \tau}{\sqrt{d}} \sum_{j=3}^{n}\left(\left\|u^{j+1}-u^{j}\right\|+\left\|u^{j}-u^{j-1}\right\|+\left\|u^{j-1}-u^{j-2}\right\|\right)\left\|q^{j+1}\right\| \\
& +\frac{5}{12} \tau \sum_{j=3}^{n}\left\|q^{j+1}\right\|\left\|q^{j-1}\right\|+\frac{1}{12} \tau \sum_{j=3}^{n}\left\|q^{j+1}\right\|\left\|q^{j-3}\right\| .
\end{aligned}
$$

A simple use of the Young's inequality yields

$$
\begin{aligned}
R H S \leq & \frac{1}{8} \tau \sum_{j=3}^{n}\left\|q^{j+1}\right\|^{2}+\frac{5}{24} \tau \sum_{j=3}^{n}\left(\left\|q^{j+1}\right\|^{2}+\left\|q^{j-1}\right\|^{2}\right) \\
& +\frac{1}{24} \tau \sum_{j=3}^{n}\left(\left\|q^{j+1}\right\|^{2}+\left\|q^{j-3}\right\|^{2}\right) \\
& +\frac{6 C_{f}^{2} C_{\mu}^{2}}{d} \tau \sum_{j=3}^{n}\left(\left\|u^{j+1}-u^{j}\right\|^{2}+\left\|u^{j}-u^{j-1}\right\|^{2}+\left\|u^{j-1}-u^{j-2}\right\|^{2}\right) .
\end{aligned}
$$

As a consequence,

$$
\begin{aligned}
R H S \leq & \frac{5}{8} \tau \sum_{j=3}^{n}\left\|q^{j+1}\right\|^{2}+\frac{18 C_{f}^{2} C_{\mu}^{2}}{d} \tau \sum_{j=3}^{n}\left\|u^{j+1}-u^{j}\right\|^{2} \\
& +\frac{12 C_{f}^{2} C_{\mu}^{2}}{d} \tau\left(\left\|u^{3}-u^{2}\right\|^{2}+\left\|u^{2}-u^{1}\right\|^{2}\right)+\frac{1}{4} \tau \sum_{j=0}^{3}\left\|q^{j}\right\|^{2} .
\end{aligned}
$$

So if $\frac{18 C_{f}^{2} C_{\mu}^{2}}{d} \tau \leq \frac{1}{2}$, i.e, $\tau \leq \tau_{0}=\frac{d}{36 C_{f}^{2} C_{\mu}^{2}}$, then we get (4.4).

\subsection{Error estimates}

Similar as in Subsection 3.3 we assume the exact solution satisfies (3.20), and $f$ satisfies (3.21). Then it can be verified that, the exact solution of (2.7) satisfies: for arbitrary $(v, r) \in V_{h} \times V_{h}$

$$
\begin{aligned}
\left(U^{n+1}, v\right)= & \left(U^{n}, v\right)+\frac{23}{12} \tau \mathcal{H}\left(f\left(U^{n}\right), v\right)-\frac{4}{3} \tau \mathcal{H}\left(f\left(U^{n-1}\right), v\right)+\frac{5}{12} \tau \mathcal{H}\left(f\left(U^{n-2}\right), v\right) \\
& +\frac{2}{3} \tau \mathcal{L}\left(Q^{n+1}, v\right)+\frac{5}{12} \tau \mathcal{L}\left(Q^{n-1}, v\right)-\frac{1}{12} \tau \mathcal{L}\left(Q^{n-3}, v\right)+\left(\zeta^{n}, v\right),
\end{aligned}
$$

for $n \geq 3$, where

$$
\left\|\zeta^{n}\right\| \leq C \tau^{4}
$$

with $C$ independent of $\tau$. And

$$
\left(Q^{n}, r\right)=\mathcal{K}\left(U^{n}, r\right), \quad \forall n .
$$

In this section, $\left(U^{n}, Q^{n}\right)=\left(U\left(x, t^{n}\right), Q\left(x, t^{n}\right)\right)$ denotes the exact solution of (2.7), which is slightly different with its meaning in Subsection 3.3 
Theorem 4.2. Under the condition of Theorem [3.2 assume $u$ is the solution of scheme (4.2), then (3.22) holds for $k \geq 1$.

Proof. For arbitrary $n$, let $\left(e_{u}^{n}, e_{q}^{n}\right)=\left(U^{n}-u^{n}, Q^{n}-q^{n}\right)=\left(\xi_{u}^{n}-\eta_{u}^{n}, \xi_{q}^{n}-\eta_{q}^{n}\right)$, where $\left(\xi_{u}^{n}, \xi_{q}^{n}\right)=\left(\pi_{h}^{-} U^{n}-u^{n}, \pi_{h}^{+} Q^{n}-q^{n}\right)$ and $\left(\eta_{u}^{n}, \eta_{q}^{n}\right)=\left(\pi_{h}^{-} U^{n}-U^{n}, \pi_{h}^{+} Q^{n}-\right.$ $Q^{n}$ ). Then subtracting (4.2) from (4.7) and by Lemma 2.2 we get the error equation: for arbitrary $(v, r) \in V_{h} \times V_{h}$

$$
\begin{aligned}
\left(\xi_{u}^{n+1}-\xi_{u}^{n}, v\right)= & \frac{23}{12} \tau \mathcal{D}\left(U^{n}, u^{n} ; v\right)-\frac{4}{3} \tau \mathcal{D}\left(U^{n-1}, u^{n-1}, v\right)+\frac{5}{12} \tau \mathcal{D}\left(U^{n-2}, u^{n-2}, v\right) \\
& +\frac{2}{3} \tau \mathcal{L}\left(\xi_{q}^{n+1}, v\right)+\frac{5}{12} \tau \mathcal{L}\left(\xi_{q}^{n-1}, v\right)-\frac{1}{12} \tau \mathcal{L}\left(\xi_{q}^{n-3}, v\right) \\
& +\left(\eta_{u}^{n+1}-\eta_{u}^{n}, v\right)+\left(\zeta^{n}, v\right)
\end{aligned}
$$

for $n \geq 3$, and

$$
\left(\xi_{q}^{n}, r\right)=\left(\eta_{q}^{n}, r\right)+\mathcal{K}\left(\xi_{u}^{n}, r\right), \quad \forall n .
$$

Taking $v=\xi_{u}^{n+1}$ in 4.10a), we get

$$
\frac{1}{2}\left\|\xi_{u}^{n+1}\right\|^{2}+\frac{1}{2}\left\|\xi_{u}^{n+1}-\xi_{u}^{n}\right\|^{2}-\frac{1}{2}\left\|\xi_{u}^{n}\right\|^{2}=\mathcal{Z}_{\mathrm{c}}+\mathcal{Z}_{\mathrm{d}}+\mathcal{Z}_{\mathrm{p}}
$$

where

$$
\begin{aligned}
& \mathcal{Z}_{\mathrm{c}}=\frac{23}{12} \tau \mathcal{D}\left(U^{n}, u^{n} ; \xi_{u}^{n+1}\right)-\frac{4}{3} \tau \mathcal{D}\left(U^{n-1}, u^{n-1} ; \xi_{u}^{n+1}\right)+\frac{5}{12} \tau \mathcal{D}\left(U^{n-2}, u^{n-2}, \xi_{u}^{n+1}\right) ; \\
& \mathcal{Z}_{\mathrm{d}}=\frac{2}{3} \tau \mathcal{L}\left(\xi_{q}^{n+1}, \xi_{u}^{n+1}\right)+\frac{5}{12} \tau \mathcal{L}\left(\xi_{q}^{n-1}, \xi_{u}^{n+1}\right)-\frac{1}{12} \tau \mathcal{L}\left(\xi_{q}^{n-3}, \xi_{u}^{n+1}\right) ; \\
& \mathcal{Z}_{\mathrm{p}}=\left(\eta_{u}^{n+1}-\eta_{u}^{n}, \xi_{u}^{n+1}\right)+\left(\zeta^{n}, \xi_{u}^{n+1}\right) .
\end{aligned}
$$

By applying the property (3.36) we can derive

$$
\begin{aligned}
\mathcal{Z}_{\mathrm{d}}= & -\frac{2}{3} \tau\left\|\xi_{q}^{n+1}\right\|^{2}-\frac{5}{12} \tau\left(\xi_{q}^{n+1}, \xi_{q}^{n-1}\right)+\frac{1}{12} \tau\left(\xi_{q}^{n+1}, \xi_{q}^{n-3}\right) \\
& +\frac{2}{3} \tau\left(\eta_{q}^{n+1}, \xi_{q}^{n+1}\right)+\frac{5}{12} \tau\left(\eta_{q}^{n+1}, \xi_{q}^{n-1}\right)-\frac{1}{12} \tau\left(\eta_{q}^{n+1}, \xi_{q}^{n-3}\right) .
\end{aligned}
$$

Then summing the energy equation (4.11) in time we obtain

$$
\underbrace{\frac{1}{2}\left\|\xi_{u}^{n+1}\right\|^{2}+\frac{1}{2} \sum_{j=3}^{n}\left\|\xi_{u}^{j+1}-\xi_{u}^{j}\right\|^{2}-\frac{1}{2}\left\|\xi_{u}^{3}\right\|^{2}+\frac{2}{3} \tau \sum_{j=3}^{n}\left\|\xi_{q}^{j+1}\right\|^{2}}_{L H S}=I+I I+I I I
$$


where

$$
\begin{aligned}
I & =\tau \sum_{j=3}^{n}\left\{\frac{23}{12} \mathcal{D}\left(U^{j}, u^{j} ; \xi_{u}^{j+1}\right)-\frac{4}{3} \mathcal{D}\left(U^{j-1}, u^{j-1} ; \xi_{u}^{j+1}\right)+\frac{5}{12} \mathcal{D}\left(U^{j-2}, u^{j-2}, \xi_{u}^{j+1}\right)\right\}, \\
I I & =\tau \sum_{j=3}^{n}\left\{-\frac{5}{12}\left(\xi_{q}^{j+1}, \xi_{q}^{j-1}\right)+\frac{1}{12}\left(\xi_{q}^{j+1}, \xi_{q}^{j-3}\right)\right\}, \\
I I I & =\sum_{j=3}^{n}\left\{\left(\eta_{u}^{j+1}-\eta_{u}^{j}, \xi_{u}^{j+1}\right)+\left(\zeta^{j}, \xi_{u}^{j+1}\right)+\tau\left(\eta_{q}^{j+1}, \frac{2}{3} \xi_{q}^{j+1}+\frac{5}{12} \xi_{q}^{j-1}-\frac{1}{12} \xi_{q}^{j-3}\right)\right\} .
\end{aligned}
$$

A direct use of Lemma 3.3 and the property [3.39) leads to

$$
\begin{aligned}
I & \leq C_{f} \tau \sum_{j=3}^{n}\left(\left\|\xi_{u}^{j}\right\|+\left\|\xi_{u}^{j-1}\right\|+\left\|\xi_{u}^{j-2}\right\|+h^{k+1}\right)\left(\left\|\left(\xi_{u}^{j+1}\right)_{x}\right\|+\sqrt{\mu h^{-1}} \llbracket \xi_{u}^{j+1} \rrbracket\right) \\
& \leq \frac{C_{f} C_{\mu}}{\sqrt{d}} \tau \sum_{j=3}^{n}\left(\left\|\xi_{u}^{j}\right\|+\left\|\xi_{u}^{j-1}\right\|+\left\|\xi_{u}^{j-2}\right\|+h^{k+1}\right)\left(\left\|\xi_{q}^{j+1}\right\|+h^{k+1}\right) \\
& \leq \varepsilon \tau \sum_{j=3}^{n}\left\|\xi_{q}^{j+1}\right\|^{2}+C_{\varepsilon} \frac{C_{f}^{2} C_{\mu}^{2}}{d} \tau \sum_{j=1}^{n}\left\|\xi_{u}^{j}\right\|^{2}+C h^{2 k+2},
\end{aligned}
$$

for arbitrary $\varepsilon$, where we have used the Young's inequality in the last inequality.

A simple using of the Cauchy-Schwarz inequality and the Young's inequality yields

$$
\begin{aligned}
I I \leq & \frac{1}{2} \tau \sum_{j=3}^{n}\left\|\xi_{q}^{j+1}\right\|^{2}+\frac{1}{4} \tau \sum_{j=0}^{3}\left\|\xi_{q}^{j}\right\|^{2} \\
I I I \leq & \varepsilon \tau \sum_{j=3}^{n}\left\|\xi_{u}^{j+1}\right\|^{2}+2 \varepsilon \tau \sum_{j=3}^{n}\left\|\xi_{q}^{j+1}\right\|^{2}+\varepsilon \tau \sum_{j=0}^{3}\left\|\xi_{q}^{j}\right\|^{2} \\
& +C_{\varepsilon} \sum_{j=3}^{n}\left(\frac{1}{\tau}\left\|\eta_{u}^{j+1}-\eta_{u}^{j}\right\|^{2}+\tau\left\|\eta_{q}^{j+1}\right\|^{2}+\frac{1}{\tau}\left\|\zeta^{j}\right\|^{2}\right) \\
& \leq \varepsilon \tau \sum_{j=3}^{n}\left\|\xi_{u}^{j+1}\right\|^{2}+2 \varepsilon \tau \sum_{j=3}^{n}\left\|\xi_{q}^{j+1}\right\|^{2}+\varepsilon \tau \sum_{j=0}^{3}\left\|\xi_{q}^{j}\right\|^{2}+C_{\varepsilon}\left(h^{2 k+2}+\tau^{6}\right),
\end{aligned}
$$

for arbitrary $\varepsilon$, where we have used the approximation property (3.28) and 4.8 in the last line.

Combining the above estimates, we get

$$
\begin{aligned}
L H S \leq & \left(\frac{1}{2}+3 \varepsilon\right) \tau \sum_{j=3}^{n}\left\|\xi_{q}^{j+1}\right\|^{2}+\left(\frac{1}{4}+\varepsilon\right) \tau \sum_{j=0}^{3}\left\|\xi_{q}^{j}\right\|^{2} \\
& +\varepsilon \tau\left\|\xi_{u}^{n+1}\right\|^{2}+C_{\varepsilon} \frac{C_{f}^{2} C_{\mu}^{2}}{d} \tau \sum_{j=1}^{n}\left\|\xi_{u}^{j}\right\|^{2}+C_{\varepsilon}\left(h^{2 k+2}+\tau^{6}\right) .
\end{aligned}
$$


Choosing $\varepsilon=\frac{1}{18}$, we get

$$
\frac{4}{9}\left\|\xi_{u}^{n+1}\right\|^{2}-\frac{1}{2}\left\|\xi_{u}^{3}\right\|^{2} \leq \frac{11}{36} \tau \sum_{j=0}^{3}\left\|\xi_{q}^{j}\right\|^{2}+\frac{C_{f}^{2} C_{\mu}^{2}}{d} \tau \sum_{j=1}^{n}\left\|\xi_{u}^{j}\right\|^{2}+C\left(h^{2 k+2}+\tau^{6}\right),
$$

if $\tau<1$. That is,

$$
\left\|\xi_{u}^{n+1}\right\|^{2} \leq C\left\|\xi_{u}^{3}\right\|^{2}+C \tau \sum_{j=0}^{3}\left\|\xi_{q}^{j}\right\|^{2}+\frac{C_{f}^{2} C_{\mu}^{2}}{d} \tau \sum_{j=1}^{n}\left\|\xi_{u}^{j}\right\|^{2}+C\left(h^{2 k+2}+\tau^{6}\right) .
$$

In what follows we give the proof for Theorem 4.2 by the induction method. First noting that $\xi_{u}^{0}=0$, hence from (4.10b) we can show

$$
\left\|\xi_{q}^{0}\right\| \leq C h^{-1}\left\|\xi_{u}^{0}\right\|+C h^{k+1}=C h^{k+1} .
$$

Then if we adopt a second or third order scheme to start the scheme (4.2), for example, we adopt the second order IMEX RK LDG scheme (3.2) to start the scheme, we can obtain

$$
\left\|\xi_{u}^{j}\right\|^{2}+\tau\left\|\xi_{q}^{j}\right\|^{2} \leq C\left(h^{2 k+2}+\tau^{6}\right), \quad \text { for } \quad j=1,2,3,
$$

if $\tau \leq \tau_{0}$, where $\tau_{0}$ is a positive constant which is independent of $h$. Note that the local truncation error for a second order time discretization scheme is of order $\mathcal{O}\left(\tau^{3}\right)$, hence the above estimate is true, but we skip the detailed proof to save space. Therefore, we have

$$
\tau \sum_{j=0}^{3}\left\|\xi_{q}^{j}\right\|^{2} \leq C\left(h^{2 k+2}+\tau^{6}\right)
$$

Then for $n=3$, from 4.15 we can get

$$
\left\|\xi_{u}^{4}\right\| \leq C\left(h^{k+1}+\tau^{3}\right)
$$

Assume (3.22) holds for all $n \leq m$, also from (4.15) we get

$$
\left\|\xi_{u}^{m+1}\right\|^{2} \leq C\left(h^{2 k+2}+\tau^{6}\right)+\frac{C_{f}^{2} C_{\mu}^{2}}{d} \tau \sum_{j=3}^{n}\left(h^{2 k+2}+\tau^{6}\right) \leq C\left(h^{2 k+2}+\tau^{6}\right)
$$

since $\frac{C_{f}^{2} C_{\mu}^{2}}{d}$ is bounded. Hence we complete the proof of this theorem.

\section{Numerical experiments}

The purpose of this section is to numerically validate the stability and error accuracy for the Runge-Kutta IMEX LDG schemes (3.2), 3.3) and the multistep IMEX LDG schemes (4.1), (4.2). For the third order Runge-Kutta IMEX LDG scheme (3.3), we take the parameter $\alpha_{1}=-0.35$ as the choice in [3]. 
In all the experiments, we take the finite element space as piecewise linear polynomials for the second order schemes (3.2) and (4.1), and piecewise 280 quadratic polynomials for the third order schemes (3.3) and (4.2), respectively. In the implementation of the second order multi-step IMEX LDG scheme (4.1), we adopt the first order IMEX RK LDG scheme (3.1) to compute the solution at the first time level. And to implement the third order multi-step IMEX LDG scheme (4.2) we use the second order IMEX RK LDG scheme (3.2) to compute the solutions at the first three time levels.

We will take the Burgers equation

$$
\begin{array}{ll}
U_{t}+\left(\frac{U^{2}}{2}\right)_{x}=d U_{x x}+g(x, t), & (x, t) \in Q_{T}=(-\pi, \pi) \times(0, T], \\
U(x, 0)=U_{0}(x), & (x, t) \in \Omega=(-\pi, \pi),
\end{array}
$$

as an example to test the stability of the four schemes. We will consider two cases:

Case (i): $U_{0}(x)=\sin (x), g(x, t)=\cos (x+t)(1+\sin (x+t))+d \sin (x+t)$, the exact solution is $u(x, t)=\sin (x+t)$.

Case (ii): $U_{0}(x)=\frac{1}{2} \sin (x), g(x, t)=\frac{1}{2} \cos (x+t)\left(1+\frac{1}{2} \sin (x+t)\right)+\frac{1}{2} d \sin (x+$ $t$ ), the exact solution is $u(x, t)=\frac{1}{2} \sin (x+t)$.

Table 2: The maximum time step $\tau_{0}$ to ensure that the $L^{2}$-norm is bounded with time for the schemes.

\begin{tabular}{|c|c|c|c|c|}
\hline & \multicolumn{2}{|c|}{ Case (i) } & \multicolumn{2}{c|}{ Case (ii) } \\
\hline scheme & $d=0.05$ & $d=0.1$ & $d=0.05$ & $d=0.1$ \\
\hline second order RK (3.2) & 0.183 & 0.327 & 0.717 & 1.156 \\
\hline third order RK [3.3 & 0.497 & 0.823 & 1.228 & 1.545 \\
\hline second order multi-step 4.1] & 0.020 & 0.048 & 0.045 & 0.113 \\
\hline third order multi-step 4.2] & 0.057 & 0.134 & 0.518 & 0.689 \\
\hline
\end{tabular}

Table 2 lists the maximum time step $\tau_{0}$ which can be chosen to ensure the stability of the schemes (in the sense that the $L^{2}$ norm of $u$ is bounded with time, if we take $\tau=\tau_{0}+0.001$, the numerical solution will blow up) for solving problem (5.1) on uniform meshes, with mesh size $h=2 \pi / N$, where $N$ is the number of cells. In this test, the final computing time is $T=5000$. We take $N=320,640,1280$ and obtain the same $\tau_{0}$. This verifies that $\tau_{0}$ is independent of the mesh size.

Even though the relationship between $\tau_{0}$ and $d / C_{f}^{2}$ is not so clean as the linear case (in 14] we can see $\tau_{0} \approx \nu d / c^{2}$, where $c$ is the convection coefficient), we can observe that for all the four schemes, $\tau_{0}$ is larger if the diffusion coefficient $d$ is bigger, but it is smaller when the maximum flow velocity is bigger. Besides, we can find that for the same type of schemes, the third order scheme admits larger time step than the second order scheme, and the IMEX RK schemes admit larger time step than the multi-step type schemes. 
Next we will test the following three examples to verify the orders of accuracy of the four schemes.

Example 1.

$$
\begin{array}{ll}
U_{t}+\left(\frac{U^{2}}{2}\right)_{x}=d U_{x x}+g(x, t), & (x, t) \in Q_{T}=(-\pi, \pi) \times(0, T], \\
U(x, 0)=\sin (x), & x \in \Omega=(-\pi, \pi),
\end{array}
$$

where $g(x, t)=\frac{1}{2} e^{-2 d t} \sin (2 x)$.

Example 2.

$$
\begin{array}{ll}
U_{t}+\left(\frac{U^{3}}{3}\right)_{x}=d U_{x x}+g(x, t), & (x, t) \in Q_{T}=(-\pi, \pi) \times(0, T], \\
U(x, 0)=\sin (x), & x \in \Omega=(-\pi, \pi),
\end{array}
$$

where $g(x, t)=e^{-3 d t} \sin ^{2}(x) \cos (x)$.

Example 3.

$$
\begin{array}{ll}
U_{t}+\left(e^{U}\right)_{x}=d U_{x x}+g(x, t), & (x, t) \in Q_{T}=(-\pi, \pi) \times(0, T], \\
U(x, 0)=\sin (x), & x \in \Omega=(-\pi, \pi),
\end{array}
$$

where $g(x, t)=e^{e^{-d t} \sin (x)} e^{-d t} \cos (x)$.

The exact solutions of (5.2), (5.3) and (5.4) are all

$$
U(x, t)=e^{-d t} \sin (x) .
$$

In the following tests, we will take $d=0.5$, and the final computing time is $T=10$.

Tables 36 are the $L^{2}$ errors and orders of accuracy for the IMEX RK LDG schemes (3.2), (3.3) and the multi-step IMEX LDG schemes (4.1), (4.2) for solv315 ing the above three examples on nonuniform meshes, respectively. The nonuniform meshes are obtained by randomly perturbing each node in the uniform mesh by up $20 \%$. We take $\tau=h$ in all the tests. We can clearly observe the designed orders of accuracy from these tables.

Table 3: The second order Runge-Kutta IMEX LDG scheme 3.2, $k=1$.

\begin{tabular}{|c|cc|cc|cc|}
\hline & \multicolumn{2}{|c|}{ Example 1 } & \multicolumn{2}{c|}{ Example 2 } & \multicolumn{2}{c|}{ Example 3 } \\
\hline$N$ & $L^{2}$ error & order & $L^{2}$ error & order & $L^{2}$ error & order \\
\hline 40 & $2.64 \mathrm{E}-05$ & - & $2.54 \mathrm{E}-05$ & - & $2.21 \mathrm{E}-05$ & - \\
80 & $6.60 \mathrm{E}-06$ & 2.00 & $6.55 \mathrm{E}-06$ & 1.96 & $5.64 \mathrm{E}-06$ & 1.97 \\
160 & $1.61 \mathrm{E}-06$ & 2.04 & $1.63 \mathrm{E}-06$ & 2.01 & $1.41 \mathrm{E}-06$ & 2.00 \\
320 & $4.00 \mathrm{E}-07$ & 2.01 & $3.99 \mathrm{E}-07$ & 2.03 & $3.52 \mathrm{E}-07$ & 2.01 \\
640 & $1.01 \mathrm{E}-07$ & 1.99 & $1.00 \mathrm{E}-07$ & 1.99 & $8.88 \mathrm{E}-08$ & 1.99 \\
\hline
\end{tabular}


Table 4: The third order Runge-Kutta IMEX LDG scheme [3.3, $k=2$.

\begin{tabular}{|c|cc|cc|cc|}
\hline & \multicolumn{2}{|c|}{ Example 1} & \multicolumn{2}{c|}{ Example 2} & \multicolumn{2}{c|}{ Example 3 } \\
\hline$N$ & $L^{2}$ error & order & $L^{2}$ error & order & $L^{2}$ error & order \\
\hline 40 & $8.18 \mathrm{E}-07$ & - & $8.45 \mathrm{E}-07$ & - & $7.43 \mathrm{E}-07$ & - \\
80 & $1.04 \mathrm{E}-07$ & 2.98 & $1.09 \mathrm{E}-07$ & 2.95 & $9.41 \mathrm{E}-08$ & 2.98 \\
160 & $1.33 \mathrm{E}-08$ & 2.97 & $1.39 \mathrm{E}-08$ & 2.98 & $1.18 \mathrm{E}-08$ & 3.00 \\
320 & $1.67 \mathrm{E}-09$ & 2.99 & $1.74 \mathrm{E}-09$ & 2.99 & $1.48 \mathrm{E}-09$ & 2.99 \\
640 & $2.09 \mathrm{E}-10$ & 3.00 & $2.18 \mathrm{E}-10$ & 3.00 & $1.85 \mathrm{E}-10$ & 3.00 \\
\hline
\end{tabular}

Table 5: The second order multi-step IMEX LDG scheme 4.1], $k=1$.

\begin{tabular}{|c|cc|cc|cc|}
\hline & \multicolumn{2}{|c|}{ Example 1} & \multicolumn{2}{c|}{ Example 2} & \multicolumn{2}{c|}{ Example 3} \\
\hline$N$ & $L^{2}$ error & order & $L^{2}$ error & order & $L^{2}$ error & order \\
\hline 40 & $9.31 \mathrm{E}-05$ & - & $9.17 \mathrm{E}-05$ & - & $4.76 \mathrm{E}-05$ & - \\
80 & $2.32 \mathrm{E}-05$ & 2.01 & $2.30 \mathrm{E}-05$ & 2.00 & $1.22 \mathrm{E}-05$ & 1.97 \\
160 & $5.83 \mathrm{E}-06$ & 1.99 & $5.80 \mathrm{E}-06$ & 1.99 & $3.09 \mathrm{E}-06$ & 1.98 \\
320 & $1.46 \mathrm{E}-06$ & 2.00 & $1.45 \mathrm{E}-06$ & 2.00 & $7.76 \mathrm{E}-07$ & 1.99 \\
640 & $3.64 \mathrm{E}-07$ & 2.00 & $3.63 \mathrm{E}-07$ & 2.00 & $1.94 \mathrm{E}-07$ & 2.00 \\
\hline
\end{tabular}

Table 6: The third order multi-step IMEX LDG scheme 4.2], $k=2$.

\begin{tabular}{|c|cc|cc|cc|}
\hline & \multicolumn{2}{|c|}{ Example 1 } & \multicolumn{2}{c|}{ Example 2} & \multicolumn{2}{c|}{ Example 3 } \\
\hline$N$ & $L^{2}$ error & order & $L^{2}$ error & order & $L^{2}$ error & order \\
\hline 40 & $1.05 \mathrm{E}-05$ & - & $1.08 \mathrm{E}-05$ & - & $7.36 \mathrm{E}-04$ & - \\
80 & $1.24 \mathrm{E}-06$ & 3.08 & $1.28 \mathrm{E}-06$ & 3.08 & $2.29 \mathrm{E}-07$ & 11.65 \\
160 & $1.53 \mathrm{E}-07$ & 3.02 & $1.57 \mathrm{E}-07$ & 3.02 & $2.99 \mathrm{E}-08$ & 2.94 \\
320 & $1.89 \mathrm{E}-08$ & 3.02 & $1.94 \mathrm{E}-08$ & 3.02 & $3.78 \mathrm{E}-09$ & 2.98 \\
640 & $2.35 \mathrm{E}-09$ & 3.01 & $2.41 \mathrm{E}-09$ & 3.01 & $4.77 \mathrm{E}-10$ & 2.99 \\
\hline
\end{tabular}




\section{Concluding remarks}

320

We consider several specific implicit-explicit time marching methods coupled with the LDG schemes for solving nonlinear convection-diffusion problems with periodic boundary conditions. Both Runge-Kutta type and multi-step type IMEX schemes are considered. By the aid of energy techniques, we prove that the corresponding IMEX LDG schemes are stable if $\tau \leq \tau_{0}$, where the constant $\tau_{0}$ is independent of the mesh size $h$. We also present optimal error estimates in both space and time, for the third order IMEX Runge-Kutta scheme and the third order multi-step IMEX scheme coupled with the LDG spatial discretization, under the same temporal condition, if a general monotone numerical flux is adopted. The extension of our analysis to multi-dimensional nonlinear convection-diffusion problems require some technical treatment to obtain the key relationship between the numerical solution and its gradient, also the extension of the error estimate from one dimension to multi-dimension is not straightforward, especially for general triangular meshes. This constitutes our ongoing work. In the future, we would like to consider the IMEX LDG method for solving nonlinear convection-diffusion problems with nonlinear diffusion.

\section{Appendix}

In this Appendix, we will give the proof of (3.42). Before doing that, we will give some preliminary work. First we follow 21] to introduce an important quantity

$$
\alpha(\hat{f} ; p) \equiv \alpha\left(\hat{f} ; p^{-}, p^{+}\right) \equiv \begin{cases}\llbracket p \rrbracket^{-1}(f(\bar{p})-\hat{f}(p)) & \text { if } \llbracket p \rrbracket \neq 0, \\ \left|f^{\prime}(\bar{p})\right| & \text { if } \llbracket p \rrbracket=0,\end{cases}
$$

for any $p \in H^{1}\left(\mathcal{T}_{h}\right)$, to measure the viscosity provided by the numerical flux, where $\bar{p}=\frac{p^{-}+p^{+}}{2}$. From the Lipschitz continuity of $\hat{f}$, we can get that $\alpha(\hat{f} ; p)$ is bounded for any $\left(p^{-}, p^{+}\right) \in \mathbb{R}^{2}$. We would like to use the notation $C_{f}$ to denote the bound of $\alpha(\hat{f} ; p)$. In addition, we would like to assume

$$
\left|\alpha\left(\hat{f} ; p_{1}\right)-\alpha\left(\hat{f} ; p_{2}\right)\right| \leq C_{f}\left(\left|\bar{p}_{1}-\bar{p}_{2}\right|+\left|\llbracket p_{1} \rrbracket\right|+\left|\llbracket p_{2} \rrbracket\right|\right),
$$

for arbitrary $p_{1}, p_{2} \in H^{1}\left(\mathcal{T}_{h}\right)$. It can be verified that this assumption is valid for several well known monotone numerical fluxes, such as the upwind flux, the Godunov flux and the Lax-Friedrichs flux.

Next we would like to follow 21] to divide the operator $\mathcal{D}(U, u ; v)$ (here and below, we omit the superscript $n, \ell$ for simplicity of notations) into three parts, namely,

$$
\mathcal{D}(U, u ; v)=\mathcal{H}^{l i n}\left(f^{\prime}(U) ; e_{u}, v\right)+\mathcal{H}^{n l s}\left(e_{u} ; v\right)+\mathcal{V}(u ; v)
$$


where

$$
\begin{aligned}
\mathcal{H}^{l i n}\left(f^{\prime}(U) ; w, v\right) & =\sum_{j=1}^{N}\left[\left(f^{\prime}(U) w, v_{x}\right)_{j}+\left(f^{\prime}(U) \bar{w} \llbracket v \rrbracket\right)_{j-\frac{1}{2}}\right], \\
\mathcal{H}^{n l s}\left(e_{u} ; v\right) & =\sum_{j=1}^{N}\left[\left(T_{i n t}\left(e_{u}\right), v_{x}\right)_{j}+\left(T_{b r y}\left(e_{u}\right) \llbracket v \rrbracket\right)_{j-\frac{1}{2}}\right], \\
\mathcal{V}(u ; v) & =\sum_{j=1}^{N}((f(\bar{u})-\hat{f}(u)) \llbracket v \rrbracket)_{j-\frac{1}{2}}=\sum_{j=1}^{N}(\alpha(\hat{f} ; u) \llbracket u \rrbracket \llbracket v)_{j-\frac{1}{2}} .
\end{aligned}
$$

Here

$$
\begin{aligned}
& T_{\text {int }}\left(e_{u}\right)=f(U)-f(u)-f^{\prime}(U) e_{u}, \\
& T_{b r y}\left(e_{u}\right)=f(U)-f(\bar{u})-f^{\prime}(U) \bar{e}_{u},
\end{aligned}
$$

340 are the nonlinear part of $f(\cdot)$ in the interior element and on the element boundary, respectively.

Lemma 7.1. For arbitrary $w, v \in V_{h}$, we have

$$
\begin{aligned}
\left|\mathcal{H}^{l i n}\left(f^{\prime}(U) ; w, v\right)\right| & \leq C_{f}\left(\|w\|+\left\|w_{x}\right\|+\sqrt{\mu h^{-1}}\|w\|\right)\|v\|, \\
\left|\mathcal{H}^{n l s}\left(e_{u} ; v\right)\right| & \leq C_{f} h^{-1}\left\|e_{u}\right\|_{\infty}\left(\left\|\xi_{u}\right\|+h^{k+1}\right)\|v\| .
\end{aligned}
$$

Proof. By (7.4a), we integrate by parts to obtain

$$
\mathcal{H}^{l i n}\left(f^{\prime}(U) ; w, v\right)=\sum_{j=1}^{N}\left[-\left(f^{\prime}(U)_{x} w, v\right)_{j}-\left(f^{\prime}(U) w_{x}, v\right)_{j}-\left(f^{\prime}(U) \bar{v} \llbracket w \rrbracket\right)_{j-\frac{1}{2}}\right] .
$$

From assumptions (3.21) and (3.25), we know that $\left|f^{\prime}(U)\right|$ and $\left|f^{\prime}(U)_{x}\right|=$ $\left|f^{\prime \prime}(U) U_{x}\right|$ are all uniformly bounded, and we still denote the bound of them by $C_{f}$. By a simple use of the Cauchy-Schwarz inequality and the inverse inequality (2.4C) we get (7.6).

The conclusion (7.7) is almost the same as that in [21], so we omit the detailed proof.

In the following we take the term $Z=\tau\left(\mathcal{D}\left(U^{n, 2}, u^{n, 2} ; v\right)-\mathcal{D}\left(U^{n, 1}, u^{n, 1} ; v\right)\right)$ as a typical term to give the estimate for $Z_{2}$, here and below $v=\mathbb{E}_{4} \xi_{u}^{n}$. It is a little tricky to get optimal error estimate by considering the difference in this form. From (7.3) we have

$$
Z=Z_{\xi}^{l i n}-Z_{\eta}^{l i n}+Z^{n l s}+Z^{v i s}
$$

where

$$
\begin{aligned}
Z_{\xi}^{l i n} & =\tau \mathcal{H}^{l i n}\left(f^{\prime}\left(U^{n, 2}\right) ; \xi_{u}^{n, 2}, v\right)-\tau \mathcal{H}^{l i n}\left(f^{\prime}\left(U^{n, 1}\right) ; \xi_{u}^{n, 1}, v\right), \\
Z_{\eta}^{l i n} & =\tau \mathcal{H}^{l i n}\left(f^{\prime}\left(U^{n, 2}\right) ; \eta_{u}^{n, 2}, v\right)-\tau \mathcal{H}^{l i n}\left(f^{\prime}\left(U^{n, 1}\right) ; \eta_{u}^{n, 1}, v\right), \\
Z^{n l s} & =\tau \mathcal{H}^{n l s}\left(e_{u}^{n, 2} ; v\right)-\tau \mathcal{H}^{n l s}\left(e_{u}^{n, 1} ; v\right) \\
Z^{v i s} & =\tau \mathcal{V}\left(u^{n, 2} ; v\right)-\tau \mathcal{V}\left(u^{n, 1} ; v\right) .
\end{aligned}
$$


We will estimate these terms one by one. First it follows from (17.6) that

$$
\begin{aligned}
Z_{\xi}^{\text {lin }} \leq & \left.C_{f} \tau \sum_{\ell=1}^{2}\left(\left\|\xi_{u}^{n, \ell}\right\|+\left\|\left(\xi_{u}^{n, \ell}\right)_{x}\right\|+\sqrt{\mu h^{-1}} \| \xi_{u}^{n, \ell}\right]\right)\|v\| \\
\leq & C_{f} \tau \sum_{\ell=1}^{2}\left\|\xi_{u}^{n, \ell}\right\|\|v\|+\frac{C_{f} C_{\mu}}{\sqrt{d}} \tau \sum_{\ell=1}^{2}\left(\left\|\xi_{q}^{n, \ell}\right\|+\left\|\eta_{q}^{n, \ell}\right\|\right)\|v\| \\
\leq & \frac{\tau}{2}\left(\left\|\xi_{u}^{n, 1}\right\|^{2}+\left\|\xi_{u}^{n, 2}\right\|^{2}\right)+\frac{\varepsilon}{2} \tau\left(\left\|\xi_{q}^{n, 1}\right\|^{2}+\left\|\xi_{q}^{n, 2}-2 \xi_{q}^{n, 1}\right\|^{2}\right) \\
& +\left(C_{f}^{2}+\frac{C_{f}^{2} C_{\mu}^{2}}{d}\right) \tau\|v\|^{2}+C h^{2 k+2} \tau,
\end{aligned}
$$

for arbitrary $\varepsilon>0$, where the second inequality holds by (3.39) and the last one by (3.28a), the Young's inequality and the triangle inequality. Notice that

$$
Z_{\eta}^{l i n}=\underbrace{\tau \mathcal{H}^{l i n}\left(f^{\prime}\left(U^{n, 2}\right)-f^{\prime}\left(U^{n, 1}\right) ; \eta_{u}^{n, 2}, v\right)}_{A_{1}}+\underbrace{\tau \mathcal{H}^{l i n}\left(f^{\prime}\left(U^{n, 1}\right) ; \eta_{u}^{n, 2}-\eta_{u}^{n, 1}, v\right)}_{A_{2}} .
$$

From (7.4a) we have

$$
\begin{aligned}
& A_{1} \leq \tau \max \left|f^{\prime}\left(U^{n, 2}\right)-f^{\prime}\left(U^{n, 1}\right)\right|\left(\mu h^{-1}\left\|\eta_{u}^{n, 2}\right\|+\sqrt{\mu h^{-1}}\left\|\eta_{u}^{n, 2}\right\|_{\Gamma_{h}}\right)\|v\|, \\
& A_{2} \leq \tau \max \left|f^{\prime}\left(U^{n, 1}\right)\right|\left(\mu h^{-1}\left\|\eta_{u}^{n, 2}-\eta_{u}^{n, 1}\right\|+\sqrt{\mu h^{-1}}\left\|\eta_{u}^{n, 2}-\eta_{u}^{n, 1}\right\|_{\Gamma_{h}}\right)\|v\|,
\end{aligned}
$$

where we have used the Cauchy-Schwarz inequality, the inverse inequalities (2.4a) and (2.4c). Then from (3.28) and the fact that $\left|f^{\prime}\left(U^{n, 2}\right)-f^{\prime}\left(U^{n, 1}\right)\right| \leq$ $C_{f}\left|U^{n, 2}-U^{n, 1}\right| \leq C_{f} \tau$, we can get

$$
A_{1}, A_{2} \leq C_{f} \tau^{2} h^{k}\|v\|
$$

Hence the Young's inequality yields

$$
Z_{\eta}^{l i n} \leq C_{f} \tau^{4} h^{2 k}+\frac{\varepsilon}{2}\|v\|^{2}
$$

for arbitrary $\varepsilon>0$.

By applying (7.7), the assumption (3.32) and the Young's inequality we have

$$
\begin{aligned}
Z^{n l s} & \leq C_{f} \tau \sum_{\ell=1}^{2} h^{-1}\left\|e_{u}^{n, \ell}\right\|_{\infty}\left(\left\|\xi_{u}^{n, \ell}\right\|+h^{k+1}\right)\|v\| \\
& \leq C_{f} C_{0} \tau \sum_{\ell=1}^{2}\left(\left\|\xi_{u}^{n, \ell}\right\|+h^{k+1}\right)\|v\| \\
& \leq \frac{\tau}{2}\left(\left\|\xi_{u}^{n, 1}\right\|^{2}+\left\|\xi_{u}^{n, 2}\right\|^{2}\right)+C_{f}^{2} C_{0}^{2} \tau\|v\|^{2}+C h^{2 k+2} \tau .
\end{aligned}
$$


At last we estimate the term $Z^{v i s}$. From (17.4c) and the fact that $\llbracket U^{n, \ell} \rrbracket_{j-\frac{1}{2}}=$ 0 , we have

$$
Z^{v i s}=\tau \sum_{j=1}^{N}\left(\alpha\left(\hat{f} ; u^{n, 2}\right) \llbracket e_{u}^{n, 2} \rrbracket \llbracket v \rrbracket-\alpha\left(\hat{f} ; u^{n, 1}\right) \llbracket e_{u}^{n, 1} \rrbracket \llbracket v \rrbracket\right)_{j-\frac{1}{2}}=Z_{\xi}^{v i s}-Z_{\eta}^{v i s}
$$

where the property of $\alpha(\cdot, \cdot)$ plays an important role, since

$$
\begin{aligned}
Z_{\xi}^{v i s} & =\tau \sum_{j=1}^{N}\left(\alpha\left(\hat{f} ; u^{n, 2}\right) \llbracket \xi_{u}^{n, 2} \rrbracket \llbracket v \rrbracket-\alpha\left(\hat{f} ; u^{n, 1}\right) \llbracket \xi_{u}^{n, 1} \rrbracket \llbracket v \rrbracket\right)_{j-\frac{1}{2}}, \\
Z_{\eta}^{v i s} & =\tau \sum_{j=1}^{N}\left(\alpha\left(\hat{f} ; u^{n, 2}\right) \llbracket \eta_{u}^{n, 2} \rrbracket \llbracket v \rrbracket-\alpha\left(\hat{f} ; u^{n, 1}\right) \llbracket \eta_{u}^{n, 1} \rrbracket \llbracket v \rrbracket\right)_{j-\frac{1}{2}} \\
& =\tau \sum_{j=1}^{N}\left(\left(\alpha\left(\hat{f} ; u^{n, 2}\right)-\alpha\left(\hat{f} ; u^{n, 1}\right)\right) \llbracket \eta_{u}^{n, 2} \rrbracket \llbracket v \rrbracket+\alpha\left(\hat{f} ; u^{n, 1}\right) \llbracket \eta_{u}^{n, 2}-\eta_{u}^{n, 1} \rrbracket \llbracket v\right)_{j-\frac{1}{2}} .
\end{aligned}
$$

A simple use of the Cauchy-Schwarz inequality and the inverse inequality (2.4c) yields

$$
\begin{aligned}
Z_{\xi}^{v i s} & \leq C_{f} \tau \sqrt{\mu h^{-1}} \sum_{\ell=1}^{2}\left\|\xi_{u}^{n, \ell}\right\|\|v\| \leq \frac{C_{f} C_{\mu}}{\sqrt{d}} \tau \sum_{\ell=1}^{2}\left(\left\|\xi_{q}^{n, \ell}\right\|+\left\|\eta_{q}^{n, \ell}\right\|\right)\|v\| \\
& \leq \frac{\varepsilon}{2} \tau\left(\left\|\xi_{q}^{n, 1}\right\|^{2}+\left\|\xi_{q}^{n, 2}-2 \xi_{q}^{n, 1}\right\|^{2}\right)+\frac{C_{f}^{2} C_{\mu}^{2}}{d} \tau\|v\|^{2}+C h^{2 k+2} \tau,
\end{aligned}
$$

where the second inequality follows from (3.39) and the last inequality holds by (3.28a , the Young's inequality and the triangle inequality.

From the assumptions (7.2) and (3.32) we have

$$
\begin{aligned}
\left|\alpha\left(\hat{f} ; u^{n, 2}\right)-\alpha\left(\hat{f} ; u^{n, 1}\right)\right| & \leq C_{f}\left(\left|\bar{u}^{n, 2}-\bar{u}^{n, 1}\right|+\left|\llbracket u^{n, 2} \rrbracket\right|+\left|\llbracket u^{n, 1} \rrbracket\right|\right) \\
& \leq C_{f}\left(\left|U^{n, 2}-U^{n, 1}\right|+\left|\bar{e}_{u}^{n, 2}\right|+\left|\bar{e}_{u}^{n, 1}\right|+\left|\llbracket e_{u}^{n, 2} \rrbracket\right|+\left|\llbracket e_{u}^{n, 1} \rrbracket\right|\right) \\
& \leq C_{f}\left(\tau+\left\|e_{u}^{n, 1}\right\|_{\infty}+\left\|e_{u}^{n, 2}\right\|_{\infty}\right) \leq C_{f}\left(\tau+C_{0} h\right),
\end{aligned}
$$

where the second inequality holds by the triangle inequality and the fact that $\bar{U}^{n, \ell}=U^{n, \ell}$ and $\llbracket U^{n, \ell} \rrbracket=0$ for $\ell=1,2$ at the element interface. So CauchySchwarz inequality, the inverse inequality (2.4c) and (3.28) lead to

$$
Z_{\eta}^{v i s} \leq C_{f} \tau\left[\left(\tau+C_{0} h\right) h^{k}+h^{k} \tau\right]\|v\| \leq \frac{\varepsilon}{2}\|v\|^{2}+C\left(h^{2 k+2} \tau^{2}+h^{2 k} \tau^{4}\right),
$$

for arbitrary $\varepsilon>0$. As a result,

$$
Z^{v i s} \leq \frac{\varepsilon}{2} \tau\left(\left\|\xi_{q}^{n, 1}\right\|^{2}+\left\|\xi_{q}^{n, 2}-2 \xi_{q}^{n, 1}\right\|^{2}\right)+\left(\frac{\varepsilon}{2}+\frac{C_{f}^{2} C_{\mu}^{2}}{d} \tau\right)\|v\|^{2}+C\left(h^{2 k+2} \tau^{2}+h^{2 k} \tau^{4}\right) .
$$


Combining the above estimates together, we get

$$
\begin{aligned}
Z \leq & \tau\left(\left\|\xi_{u}^{n, 1}\right\|^{2}+\left\|\xi_{u}^{n, 2}\right\|^{2}\right)+\varepsilon \tau\left(\left\|\xi_{q}^{n, 1}\right\|^{2}+\left\|\xi_{q}^{n, 2}-2 \xi_{q}^{n, 1}\right\|^{2}\right) \\
& +\left(C_{f}^{2} \tau+C_{f}^{2} C_{0}^{2} \tau+\frac{C_{f}^{2} C_{\mu}^{2}}{d} \tau+\varepsilon\right)\|v\|^{2}+C\left(h^{2 k+2} \tau+h^{2 k} \tau^{4}\right) .
\end{aligned}
$$

Similar result can be obtained for $\tau\left(\mathcal{D}\left(U^{n, 3}, u^{n, 3} ; v\right)-\mathcal{D}\left(U^{n, 1}, u^{n, 1} ; v\right)\right)$. Thus we are led to (3.42).

\section{Acknowledgements}

The research of the first and third authors is supported by NSFC grant 355 11271187. The research of the second author is supported by DOE grant DEFG02-08ER25863 and NSF grant DMS-1418750.

\section{References}

[1] U. M. Ascher, S. J. Ruuth And R. J. Spiteri, Implicit-explicit RungeKutta methods for time-dependent partial differential equations, Appl. Numer. Math., 25 (1997), pp. 151-167.

[2] F. Bassi And S. ReBAY, A high-order accurate discontinuous finite element method for the numerical solution of the compressible Navier-Stokes equations, J. Comput. Phys. 131 (1997), pp. 267-279.

[3] M. P. Calvo, J. DE Frutos And J. Novo, Linearly implicit Runge-Kutta methods for advection-reaction-diffusion equations, Appl. Numer. Math., 37 (2001), pp. 535-549.

[4] P. Castillo, B. Cockburn, I. Perugia and D. Schötzau, An a priori error analysis of the local discontinuous Galerkin method for elliptic problems, SIAM J. Numer. Anal., 38 (2000), pp. 1676-1706.

[5] P. Castillo, B. Cockburn, D. Schötzau and C. Schwab, Optimal a priori error estimates for the hp-version of the local discontinuous Galerkin method for convection-diffusion problems, Math. Comp., 71 (2001), pp. 455-478.

[6] P. G. Ciarlet, The Finite Element Method for Elliptic Problems, NorthHolland, Amsterdam, New York, 1978.

[7] B. Cockburn, G. Kanschat, D. Schötzau and C. Schwab, Local discontinuous Galerkin methods for the Stokes system, SIAM J. Numer. Anal., 40 (2002), pp. 319-343.

[8] B. Cockburn and C.-W. Shu, The local discontinuous Galerkin method for time-dependent convection-diffusion systems, SIAM J. Numer. Anal., 35 (1998), pp. 2440-2463. 
[9] B. Cockburn And C.-W. Shu, Runge-Kutta discontinuous Galerkin methods for convection-dominated problems, J. Sci. Comput., 16 (2001), pp. $173-261$.

[20] Q. Zhang And C.-W. Shu, Error estimates to smooth solution of RungeKutta discontinuous Galerkin methods for scalar conservation laws, SIAM J. Numer. Anal., 42 (2004), pp. 641-666. 
[21] Q. Zhang And C.-W. Shu, Stability analysis and a priori error estimates to the third order explicit Runge-Kutta discontinuous Galerkin method for scalar conservation laws, SIAM J. Numer. Anal., 48 (2010), pp. 1038-1063. 